\title{
Low-dose dasatinib rescues cardiac function in Noonan syndrome
}

\author{
Jae-Sung Yi, ${ }^{1}$ Yan Huang, ${ }^{2}$ Andrea T. Kwaczala, ${ }^{3}$ Ivana Y. Kuo, ${ }^{1}$ Barbara E. Ehrlich, ${ }^{1}$ \\ Stuart C. Campbell, ${ }^{3}$ Frank J. Giordano, ${ }^{2}$ and Anton M. Bennett ${ }^{1,4}$ \\ ${ }^{1}$ Department of Pharmacology, ${ }^{2}$ Department of Internal Medicine, ${ }^{3}$ Department of Biomedical Engineering, ${ }^{4}$ Program in \\ Integrative Cell Signaling and Neurobiology of Metabolism, Yale School of Medicine, New Haven, Connecticut, USA
}

\begin{abstract}
Noonan syndrome (NS) is a common autosomal dominant disorder that presents with short stature, craniofacial dysmorphism, and cardiac abnormalities. Activating mutations in the PTPN11 gene encoding for the Src homology $2(\mathrm{SH} 2)$ domain-containing protein tyrosine phosphatase-2 (SHP2) causes approximately $50 \%$ of NS cases. In contrast, NS with multiple lentigines (NSML) is caused by mutations that inactivate SHP2, but it exhibits some overlapping abnormalities with NS. Protein zero-related (PZR) is a SHP2-binding protein that is hyper-tyrosyl phosphorylated in the hearts of mice from NS and NSML, suggesting that PZR and the tyrosine kinase that catalyzes its phosphorylation represent common targets for these diseases. We show that the tyrosine kinase inhibitor, dasatinib, at doses orders of magnitude lower than that used for its anticancer activities inhibited PZR tyrosyl phosphorylation in the hearts of NS mice. Low-dose dasatinib treatment of NS mice markedly improved cardiomyocyte contractility and functionality. Remarkably, a low dose of dasatinib reversed the expression levels of molecular markers of cardiomyopathy and reduced cardiac fibrosis in NS and NSML mice. These results suggest that PZR/SHP2 signaling is a common target of both NS and NSML and that low-dose dasatinib may represent a unifying therapy for the treatment of PTPN11-related cardiomyopathies.
\end{abstract}

Conflict of interest: The authors have declared that no conflict of interest exists.

Submitted: August 17, 2016 Accepted: November 1, 2016 Published: December 8, 2016

Reference information: JCI Insight. 2016;1(20):e90220 doi:10.1172/jii.insight.90220.

\section{Introduction}

Germline missense mutations in components of the Ras/MAPK pathway cause a group of autosomal dominant syndromes referred to as RASopathies. Noonan syndrome (NS, OMIM 163950) and NS with multiple lentigines (NSML, OMIM 151100) constitute two forms of RASopathies (1, 2). NS represents approximately 1:2,000 live births (3), whereas the frequency of NSML is unknown. NS and NSML share some common phenotypic features that include fasciocutaneous anomalies, developmental retardation, and cardiac defects, such as pulmonary stenosis (PS) and hypertrophic cardiomyopathy (HCM), albeit with different frequencies of occurrence. Germline mutations in the PTPN11 gene that encodes for the Src homology 2 (SH2) domain-containing protein tyrosine phosphatase-2 (SHP2) account for up to 50\% of NS and $90 \%$ of NSML cases $(4,5)$. PTPN11 mutations that cause NS most frequently present with PS $(\sim 80 \%)$, with a smaller fraction of these patients presenting with HCM $(\sim 20 \%)$. In contrast, patients with NSML feature HCM as their predominant congenital heart defect (CHD) ( 80\%). Although PS is the classical myocardial manifestation of NS, nonhypertrophic restrictive and dilated cardiomyopathies have been reported (6-10), progression from hypertrophic to congestive cardiomyopathy also occurs $(10,11)$, and heart failure is an etiology of death in NS patients (12). Identifying core signaling components utilized by both NS- and NSML-related disorders could offer new insight into the progression of these diseases and potentially suggest novel therapeutic strategies to treat the more severe manifestations of NS. The identification of therapeutic approaches that can target both NS- and NSML-related cardiomyopathies as a unified therapy will provide a novel clinical utility, given the apparent challenge in diagnosis between NS and NSML, particularly within the first year of life $(13,14)$.

SHP2 acts primarily as a positive regulator of cell signaling and in virtually all cases its catalytic activity is required for signal propagation (15). SHP2 is dynamically regulated between an "open" active and a "closed" inactive conformation (16). Engagement of upstream phosphotyrosyl peptides with the $\mathrm{NH}_{2}$ terminus SH2 domains results in a conformational change that destabilizes the "closed" conformation and promotes the "open" state, thereby activating the phosphatase (17). In addition to the SH2 domains serving 
to activate SHP2 (17), targeting of SHP2 to its upstream substrates through SH2 domain binding also is critical for signal propagation (17-19). Accordingly, NS-associated SHP2 mutants that are crippled in their ability to localize via their SH2 domains fail to manifest pathophysiological signaling (18). PTPN11-associated SHP2 mutations exist in an "open" conformation $(20,21)$. Thus, both NS and NSML mutants are endowed with enhanced binding capacity through their SH2 domains to upstream receptor and adaptor proteins, which results in the acquisition of "gain-of-function" properties. Indeed, SHP2 has been shown to function as an adaptor protein and signal independently of its phosphatase activity $(22,23)$. Therefore, the "open" conformation of SHP2 incurred through either NS or NSML mutations could provide a core mechanistic property that explains, at least in part, the overlapping clinical presentation of these diseases.

Albeit a much rarer occurrence, NS patients that do develop HCM and particularly those who do so in infancy can suffer significant consequences, including early mortality. Adults with NS require long-term cardiac follow-up, given that a third have cardiac disorders that require either drugs for heart failure or arrhythmias, or have a defibrillator or pacemaker implanted (1). Given the clinical importance there has been significant interest in developing pharmacological treatments for cardiomyopathies in NS and NSML (24). It has been shown that inhibitors of the Akt/mTOR pathway can ameliorate HCM in the NSML model $(25,26)$, whereas, genetic interference with, or pharmacologic inhibition of, MEK/ERK in a NS mouse model suppresses the development of CHD (27-30). Uncovering targets and subsequently viable pharmacological therapies that encompass the overlapping clinical spectra between NS and NSML should fulfill a significant unmet clinical need.

We have previously reported that the transmembrane glycoprotein protein zero-related (PZR) is hyper-tyrosyl phosphorylated during embryogenesis in Ptpn11 ${ }^{D 61 G /+} \mathrm{NS}$ mutant mice and postdevelopmentally in the hearts of NS and Ptpn11 $1279 \mathrm{C} /++\mathrm{NSML}$ mutant mice $(31,32)$. PZR is a transmembrane glycoprotein that is abundant in the heart and binds directly to SHP2 through its immunoreceptor tyrosine-based inhibitory motifs (ITIMs) $(33,34)$. PZR is involved in cytoskeleton-mediated signaling to ERK1/2 (32) and, in some cases, cell migration and adhesion (32, 35-37). Both SHP2-associated NS and NSML mutants induce increased SHP2 complex formation with PZR, resulting in aberrant SHP2 membrane proximity and downstream signaling (31). The identification of a PZR/SHP2 complex as a common dysregulated signaling scaffold in the hearts of both NS and NSML led to the implementation of a pharmacological approach to interfere with this complex. Here, we found that dasatinib, an FDA-approved tyrosine kinase inhibitor used for the treatment of chronic myeloid leukemia (CML), at a dramatically lower dose than that used for the treatment of CML, prevented PZR hyper-tyrosyl phosphorylation and selectively improved NS-associated cardiac dysfunction. Specifically, impaired $\mathrm{Ca}^{2+}$-mediated sarcomeric contraction coupling was improved in the cardiomyocytes from low-dose dasatinib administered NS mice, providing a direct explanation for the improved cardiac functionality. Our data further show that parameters of cardiomyopathy and fibrosis in both NS and NSML are reversed by low-dose dasatinib treatment. These data raise the possibility that low-dose dasatinib may serve as a novel therapeutic strategy for PTPN11-related cardiomyopathies.

\section{Results}

PZR tyrosyl phosphorylation in NS mice. A heterozygous knockin mouse for the Ptpn11 D61G allele has been shown to display major phenotypic features associated with NS, such as growth retardation, craniofacial dysmorphism, cardiac defects, and cognitive disability $(38,39)$. The D61G allele is a potent catalytically active Ptpn11 variant (40), and mice harboring this allele exhibit approximately $50 \%$ embryonic lethality on a 129S4/SvJae $\times$ C57BL/6J mixed background (38). It is likely that the penetrance of the D61G allele is influenced by genetic background as a result of existing modifier alleles (38). In an attempt to circumvent this lethality, and thus provide a more viable model to study the D61G allele postdevelopmentally, we crossed Ptpn11 ${ }^{\text {D61G/+ }}$ male mice (B6129SF2/Tac background) with WT B6129SF1/Tac female mice (the first filial generation from C57BL/6NTac $\times 129 \mathrm{~S} 6 / \mathrm{SvEvTac})$. We found that on this genetic background heterozygotic lethality conferred by the Ptpn $11^{D 61 G /+}$ allele was completely abrogated, as evidenced by an equivalent number of viable 3-week-old WT and Ptpn11 ${ }^{\text {D61G/+ }}$ progeny (Supplemental Table 1; supplemental material available online with this article; doi:10.1172/jci.insight.90220DS1). We confirmed that the improvement in viability of these Ptpn11 $11^{D 61 G /+}$ mice was not due to alterations in the expression of SHP2 (Figure 1A). These particular mice will be referred to as NS mice herein.

The transmembrane glycoprotein PZR is a direct SHP2-binding partner that mediates cell adhesion, migration, and conversion/extension movements in zebrafish $(31-33,41)$. The SH2 domains of SHP2 
A
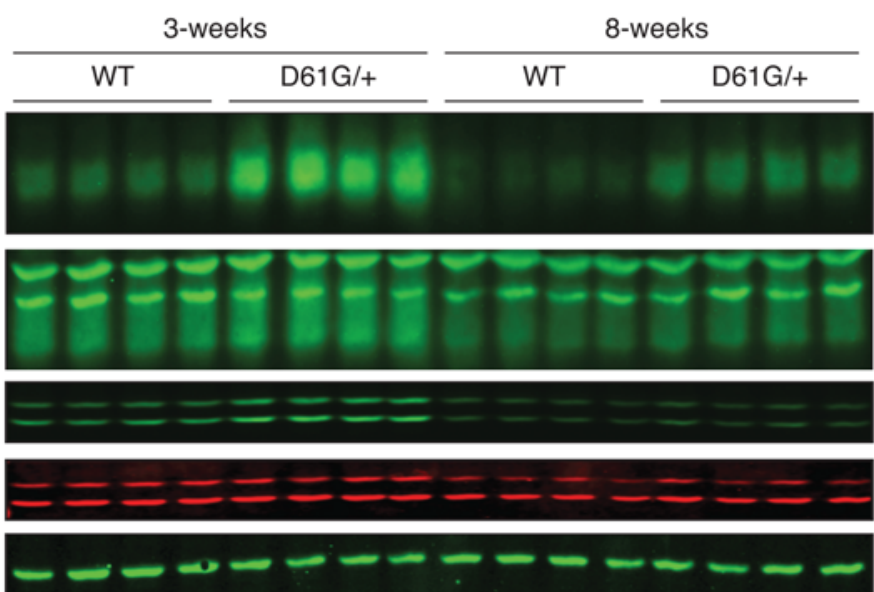

p-PZR

(Y263)

PZR

p-ERK $1 / 2$

ERK1/2

SHP2
B

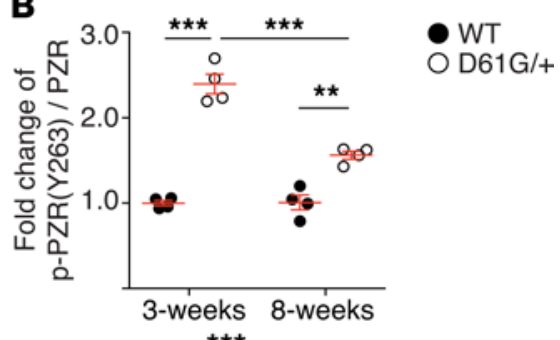

C
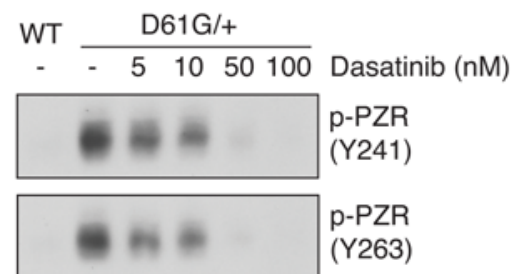

p-PZR

(Y241)

p-PZR

(Y263)

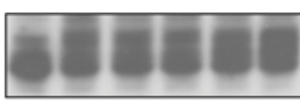

PZR

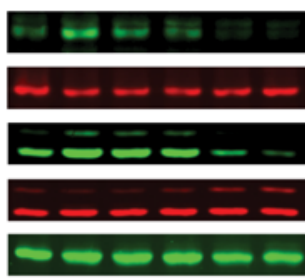

p-Src (Y416)

Src

p-ERK $1 / 2$

ERK $1 / 2$

SHP2

E
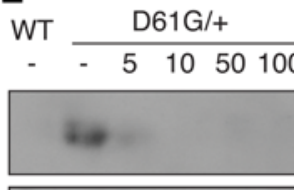

a. -600

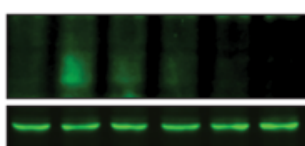

Dasatinib (nM)

PZR IP SHP2

SHP2

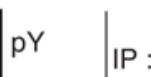

SHP2
D
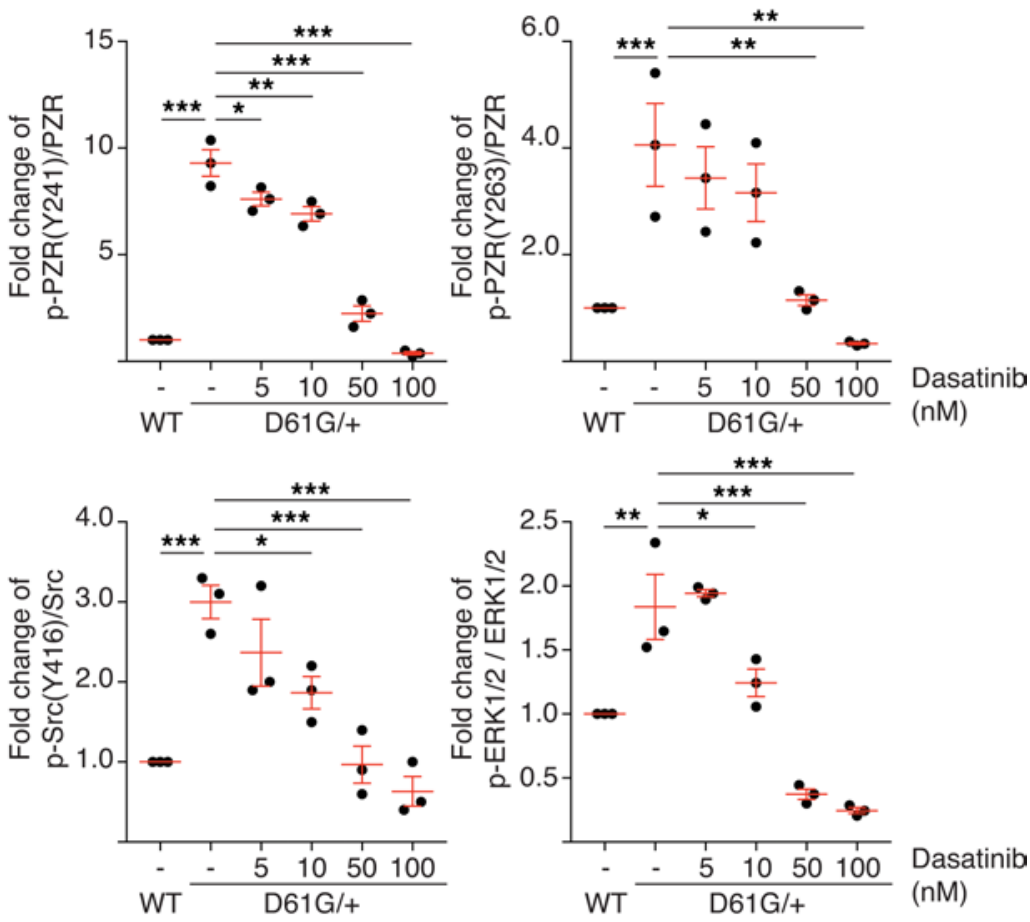

Figure 1. NS-induced PZR phosphorylation and the effects of dasatinib. (A) Heart lysates from WT and NS mice (D61G/+) were immunoblotted with anti-p-PZR (Y263), PZR, p-ERK1/2, ERK1/2, and SHP2 antibodies. (B) The quantitated ratios of p-PZR (Y263) to PZR and pERK1/2 to p-ERK1/2 from A normalized to the 3-week-old WT group ( $n=4$ per group). (C) Mouse embryonic fibroblasts from WT and NS mice were incubated with vehicle or dasatinib at the indicated concentrations for 18 hours. Whole-cell lysates were immunoblotted with anti-p-PZR (Y241), p-PZR (Y263), PZR, p-Src (Y416), Src, p-ERK1/2, ERK1/2, and SHP2 antibodies. (D) The quantitated ratios of p-PZR (Y241) to PZR, p-PZR (Y263) to PZR, p-Src (Y416) to c-Src, and p-ERK1/2 to ERK1/2 from 3 independent experiments normalized to vehicle-treated WT cells. (E) PZR and SHP2 complexes were detected by immunoprecipitation (IP) with anti-SHP2 antibodies followed by immunoblotting with anti-PZR and anti-phosphotyrosine antibodies. Immune complexes were immunoblotted with anti-SHP2 antibodies as control. Data represent mean \pm SEM and were analyzed by either 2-way (B) or 1-way ANOVA and Tukey's multiple comparison test (D). ${ }^{*} P<0.05 ;{ }^{* *} P<0.01 ;{ }^{* *} P<0.001$. 
interact directly with the tyrosyl-phosphorylated ITIMs of PZR, resulting in SHP2 activation and initiation of adhesion signaling to ERK1/2 (32). Previously, we demonstrated using a nonbiased differential phosphotyrosyl proteomic screen that PZR is the most hyper-tyrosyl-phosphorylated protein in the hearts of Ptpn11 $11^{D 61 G /+}$ mice (31). We further showed that the expression of NS- and NSML-associated SHP2 mutants is sufficient to enhance PZR tyrosyl phosphorylation and SHP2 binding (31). These data suggest that the tyrosine kinase(s) that mediates PZR tyrosyl phosphorylation and subsequently SHP2 binding to PZR may serve as an important driver to promote cardiac dysfunction in both NS and NSML. To test whether hyper-tyrosyl phosphorylation of PZR was involved in the pathogenesis of NS, we sought to pharmacologically disrupt PZR/SHP2 binding by inhibiting the PZR tyrosine kinase. As observed previously (31), PZR was hyper-tyrosyl phosphorylated on its ITIM at pY263 in the hearts of these NS mice at 3 weeks of age concomitant with elevated ERK1/2 levels as compared with WT mice (Figure 1, A and B). In 8-week-old mice, PZR remained hyper-tyrosyl phosphorylated, whereas ERK1/2 activity declined, suggesting that PZR tyrosyl phosphorylation correlates with the early activation of ERK1/2 (Figure 1, A and B).

Dasatinib inhibits PZR hyper-tyrosyl phosphorylation in NS mice. In order to probe the relevance of PZR tyrosyl phosphorylation and, hence, PZR/SHP2 signaling, we sought to inhibit PZR tyrosyl phosphorylation in NS mice. We hypothesized that if PZR tyrosyl phosphorylation and/or the tyrosine kinase responsible for its phosphorylation was important for NS-mediated cardiac anomalies, then impairing PZR tyrosyl phosphorylation should ameliorate the pathophysiological effects of the NS mutant. We tested dasatinib, an FDA-approved chemotherapeutic drug used for the treatment of CML that has broad tyrosine kinase inhibitory activity (42), for its effect on PZR tyrosyl phosphorylation. Treatment of NS-derived mouse embryo fibroblasts with dasatinib inhibited PZR hyper-tyrosyl phosphorylation at ITIMs representing pY241 and pY263 in addition to ERK1/2 and c-Src phosphorylation in a dose-dependent manner (Figure $1, \mathrm{C}$ and D). Inhibition of PZR tyrosyl phosphorylation by dasatinib also resulted in disruption of PZR/ SHP2 binding (Figure 1E). These data demonstrate that dasatinib is capable of reducing NS-associated SHP2 mutant PZR hyper-tyrosyl phosphorylation and enhanced PZR/SHP2 complex formation.

Next, we tested the effects of dasatinib to inhibit PZR tyrosyl phosphorylation in NS mice (Figure 2A). In 3-week-old NS mice, administration of dasatinib for 24 hours inhibited PZR tyrosyl phosphorylation in a dose-dependent manner (Figure 2, B and C). Remarkably, we found that PZR tyrosyl phosphorylation was significantly inhibited at doses as low as $0.1 \mathrm{mg} / \mathrm{kg}$, representing an approximate 200-fold lower dose than that used clinically for the treatment of CML (42). Next, we tested chronic low-dose dasatinib administration (Figure 2D). These experiments showed that daily injections of dasatinib $(0.1 \mathrm{mg} / \mathrm{kg})$ for 10 days reduced the levels of PZR tyrosyl phosphorylation and c-Src and ERK1/2 phosphorylation as compared with vehicle-treated NS mice (Figure 2, E and F). These data demonstrate that dasatinib can target the PZR/SHP2 pathway in the hearts of NS mice at substantially lower doses than that used for chemotherapeutic intervention.

Cardiomyopathy and cardiac dysfunction in NS mice. Postdevelopmental assessment of NS mice revealed that the heart-weight-to-body-weight ratio was significantly increased in 3-week-old NS mice as compared with WT mice (Figure 3A). Echocardiographic analysis showed a significant increase in left ventricular posterior wall thickness (LVPW) and a decrease in the left ventricular inner dimension (LVID) in NS mice as compared with WT mice (Figure 3B and Supplemental Table 2). A significant increase in the left ventricular mass was also observed in NS mice as compared with WT mice (Supplemental Table 2). However, there were no differences in either the ejection fraction (EF) or fractional shortening (FS) (Figure 3B). These results indicate that at 3 weeks of age these NS mice present with HCM without impairment of cardiac functionality. By 8 weeks of age, NS mice maintained a significant increase in their heart-weightto-body-weight ratio (Figure 3C and Supplemental Table 2). Moreover, echocardiographic analysis showed that these NS mice had reduced LVPW and an enlarged LVID in systole (Figure 3D). By 8 weeks of age, NS mice developed systolic dysfunction, as indicated by a significant reduction in both EF and FS (Figure 3D and Supplemental Table 2). These results demonstrate that HCM occurs in NS mice postdevelopmentally, with evidence of deteriorating cardiac function similar to that of some NS patients $(6,11,43,44)$.

Low-dose dasatinib treatment selectively rescues cardiac dysfunction in NS mice. To investigate the ability of low-dose dasatinib to interfere with the pathophysiological progression of NS-associated cardiac dysfunction, we first administered dasatinib to pregnant female mice carrying NS offspring (Supplemental Figure 1A). Dasatinib was administered at $0.1,0.5$, or $1.0 \mathrm{mg} / \mathrm{kg}$ daily i.p. to WT pregnant mice intercrossed with NS mice, beginning at embryonic day 7 until P9 (in nursing females). Although dasatinib treatment at 0.5 and $1.0 \mathrm{mg} / \mathrm{kg} / \mathrm{d}$ showed embryonic lethality, at $0.1 \mathrm{mg} / \mathrm{kg} / \mathrm{d}$ the expected Mendelian ratio was obtained 
A

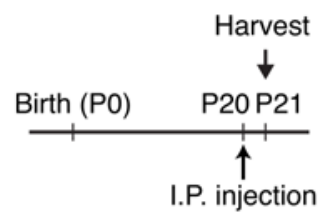

$(0.1,0.5$ or $1.0 \mathrm{mg} / \mathrm{kg}$ )

B

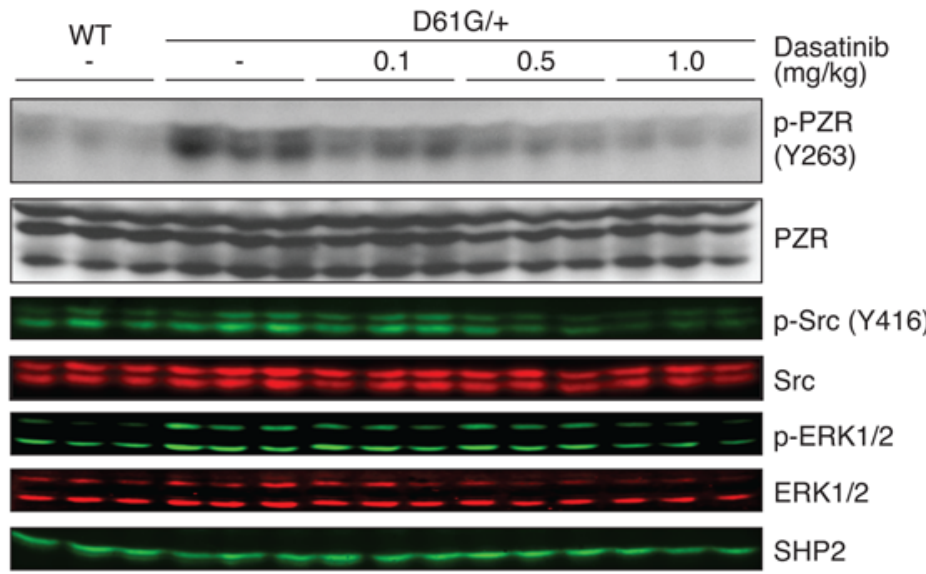

C

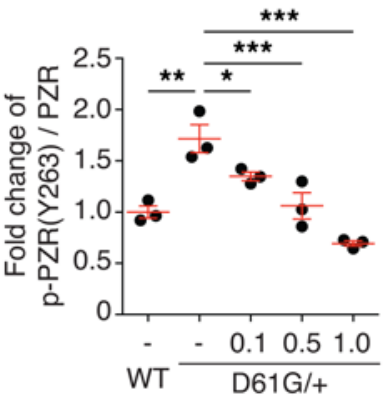

D
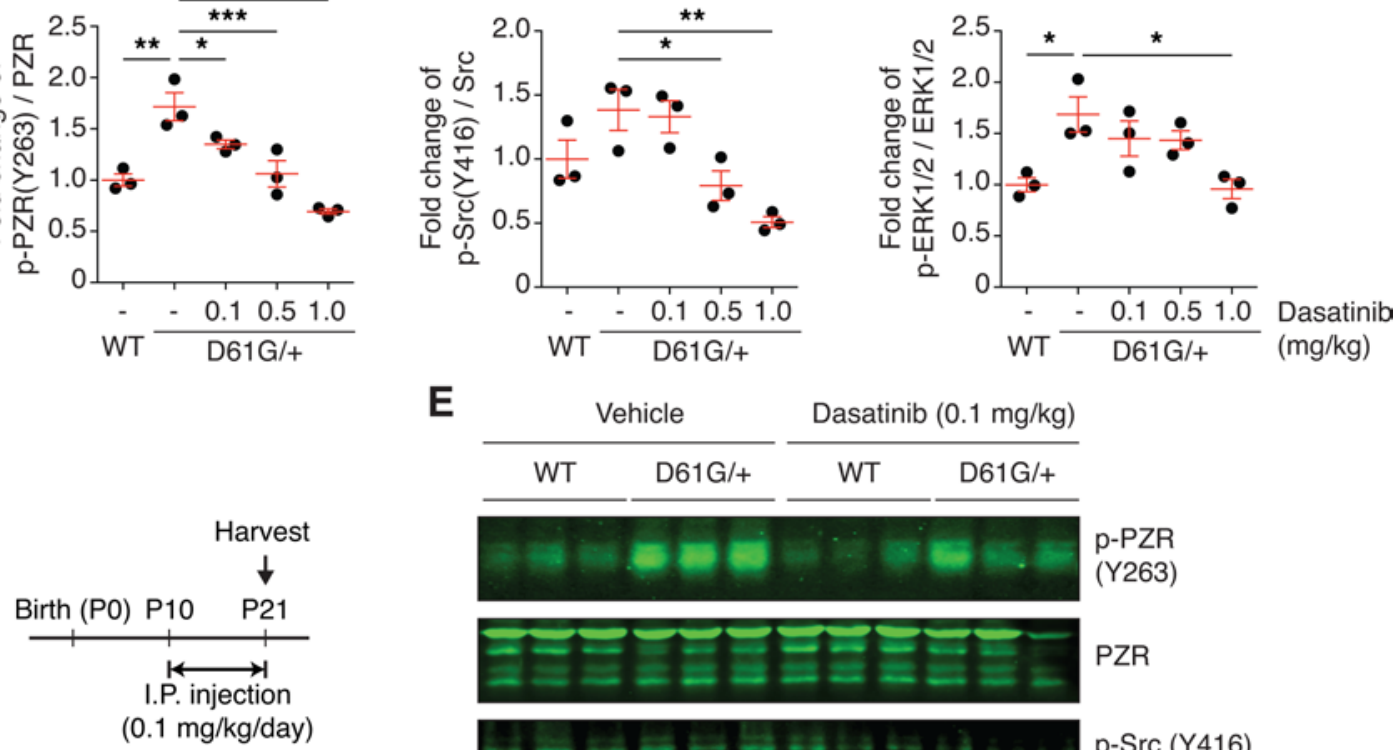

E

\begin{tabular}{|c|c|c|c|}
\hline \multicolumn{2}{|c|}{ Vehicle } & \multicolumn{2}{|c|}{ Dasatinib $(0.1 \mathrm{mg} / \mathrm{kg}$} \\
\hline WT & $\mathrm{D} 61 \mathrm{G} /+$ & WT & D61G/+ \\
\hline
\end{tabular}

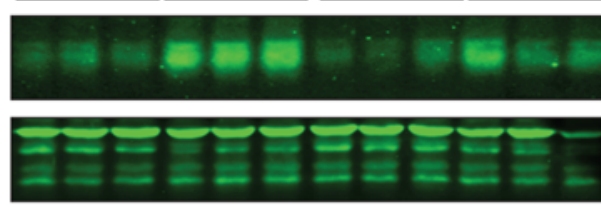

p-PZR

(Y263)

PZR

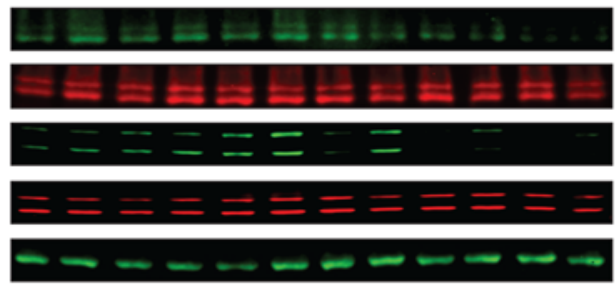

p-Src (Y416)

Src

p-ERK $1 / 2$

ERK1/2

SHP2

$\mathbf{F}$

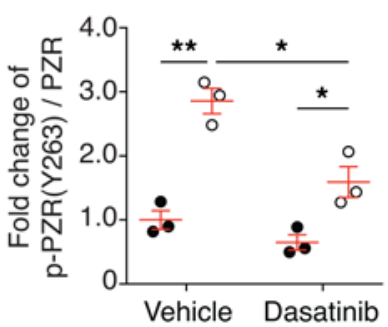

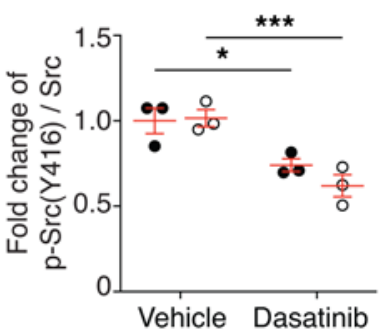

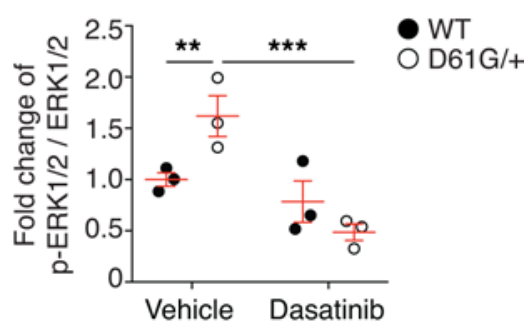

Figure 2. Dasatinib inhibits PZR hyper-tyrosyl phosphorylation in the hearts of NS mice. (A) Schematic of single-dose dasatinib administration. Vehicle or dasatinib $(0.1,0.5$, or $1.0 \mathrm{mg} / \mathrm{kg}$ ) was i.p. injected into WT and NS mice (D61G/+) at P20. The heart was isolated 24 hours after administration. (B) Heart lysates were immunoblotted with p-PZR (Y263), PZR, p-Src (Y416), Src, p-ERK1/2, ERK1/2, and SHP2 antibodies. (C) The ratio of p-PZR (Y263) to PZR, p-Src (Y416) to c-Src, and p-ERK1/2 to ERK1/2 normalized to the vehicle-injected WT group ( $n=3$ per group). (D) Schematic of chronic dasatinib administration. Vehicle or dasatinib $(0.1 \mathrm{mg} / \mathrm{kg})$ was injected i.p. into WT and NS mice from P10 to P21. (E) Heart lysates were immunoblotted with p-PZR (Y263), PZR, p-Src (Y416), Src, p-ERK1/2, ERK1/2, and SHP2 antibodies. (F) The ratio of p-PZR (Y263) to PZR, p-Src (Y416) to c-Src, and $\mathrm{p}$-ERK1/2 to ERK1/2 normalized to the vehicle-injected WT group ( $n=3$ per group). Data represent mean \pm SEM and were analyzed with 1 -way (C) or 2-way (F) ANOVA and Tukey's multiple comparison test. ${ }^{*} P<0.05 ;{ }^{*} P<0.01 ;{ }^{* * *} P<0.001$. 
A

B
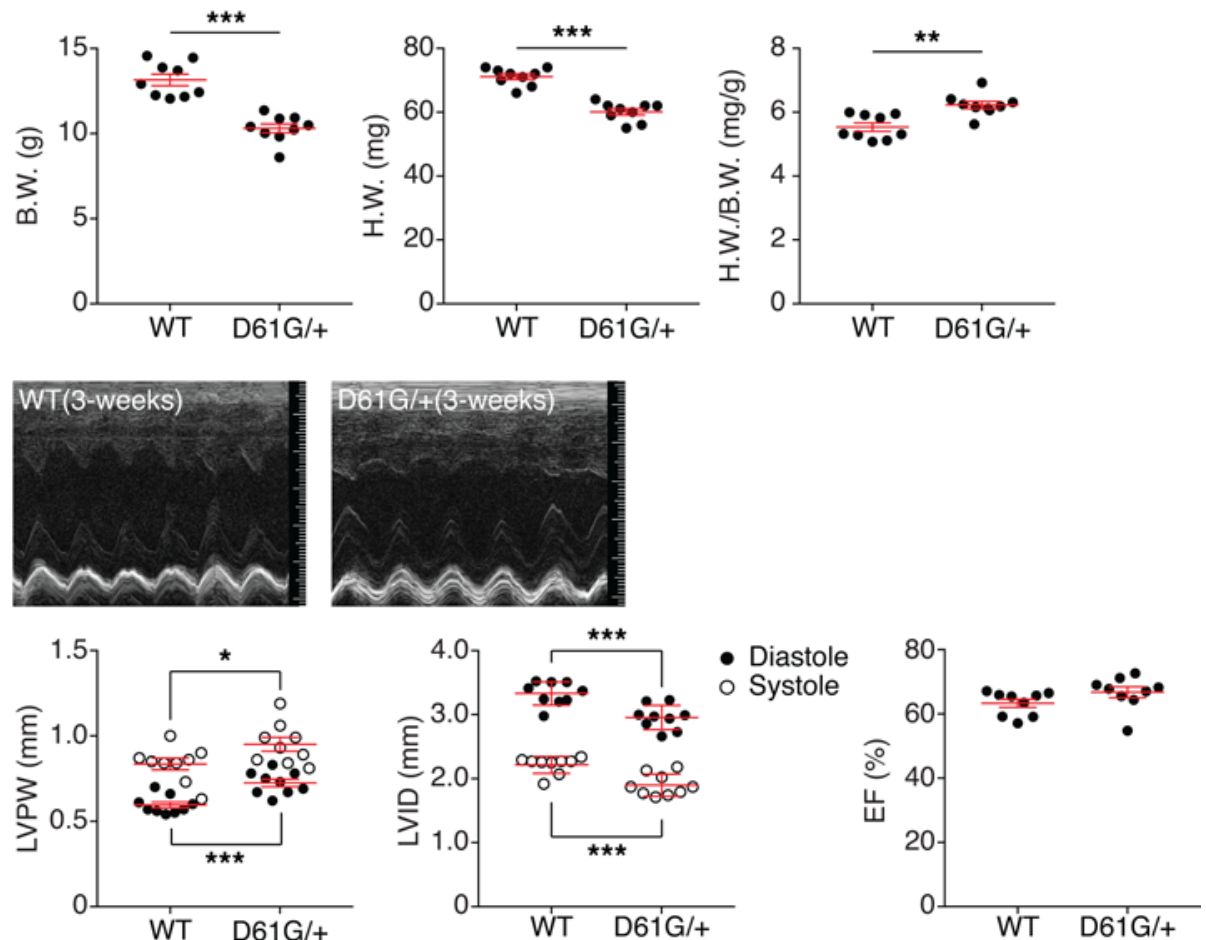

C
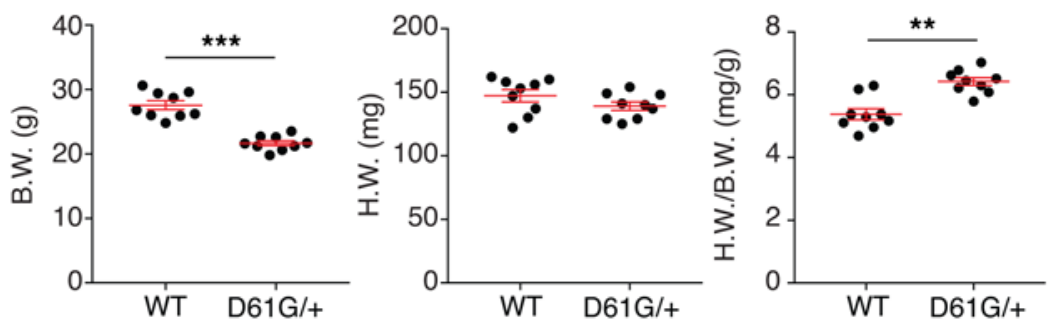

D
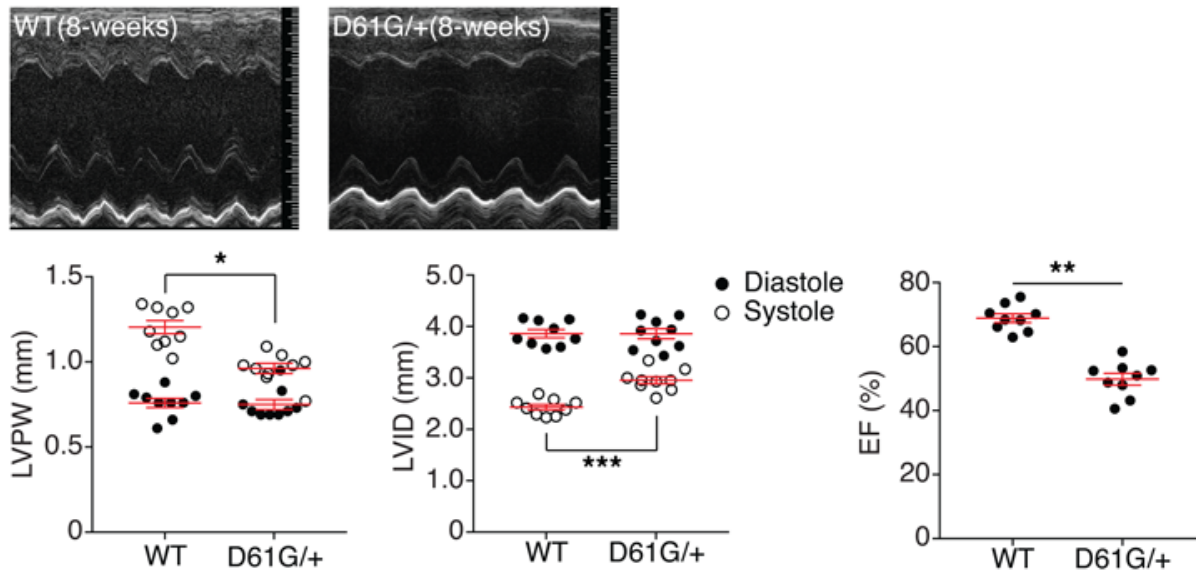

Figure 3. Cardiomyopathy and cardiac dysfunction in NS mice. (A) Body weight (B.W.), heart weight (H.W.), and the ratio of H.W. to B.W. were measured from WT and NS mice (D61G/+) at 3 weeks ( $n=9$ per genotype). (B) WT and NS mouse hearts were analyzed by echocardiography at 3 weeks. Representative echocardiograms of 3-week-old mouse hearts. Left ventricular posterior wall thickness (LVPW), left ventricular inner dimensions (LVID) in diastole and systole, and ejection fraction (\%EF) of 3 -week-old mouse hearts were measured ( $n=9$ per genotype). (C) B.W., H.W., and the ratio of H.W. to B.W. were measured from WT and NS mice at 8 weeks ( $n=9$ per genotype). (D) WT and NS mouse hearts were analyzed by echocardiography at 8 weeks. Representative echocardiograms of 8-week-old mouse hearts. LVPW, LVID in diastole and systole, and \%EF of 3-week-old mouse hearts were measured $(n=9$ per genotype). Data represent mean \pm SEM and were analyzed with unpaired Student's $t$ test. ${ }^{*} P<0.05$; ${ }^{*} P<0.01$; ${ }^{* * *} P<0.001$

(Supplemental Table 3). After P10, dasatinib injections were resumed directly in individual pups daily until 6 weeks (P42) after birth (Supplemental Figure 1A) WT mice treated with either vehicle or dasatinib showed similar body weight, as did NS mice (Supplemental Figure 1B). Cardiac function of NS mice at 6 weeks of age showed that the EF and FS in vehicle-treated NS mice were significantly reduced as compared with vehicle-treated WT mice (Supplemental Table 4). Notably, dasatinib-treated NS mice at P42 exhibited values of $\mathrm{EF}$ and $\mathrm{FS}$ that returned to levels comparable to those of both treated and untreated WT mice (Supplemental Table 4). These data indicate that dasatinib treatment in utero can rescue the impaired cardiac function observed in NS mice.

Although dasatinib when administered in utero yielded negligible

signs of lethality (Supplemental Table 3), a more compelling test of dasatinib's effectiveness would be to determine its effect on cardiac function when administered postdevelopmentally in NS mice. Therefore, we determined whether dasatinib improved cardiac dysfunction postdevelopmentally (Figure 4A). NS mice on this background were found to exhibit reduced growth, facial dysmorphism, and splenomegaly similar to that reported previously (38) (Supplemental Figure 2-4). Postnatal dasatinib $(0.1 \mathrm{mg} / \mathrm{kg}$ ) treatment did not improve any of these NS-related pathologies (Supplemental Figure 2-4). Moreover, no evidence of 
A

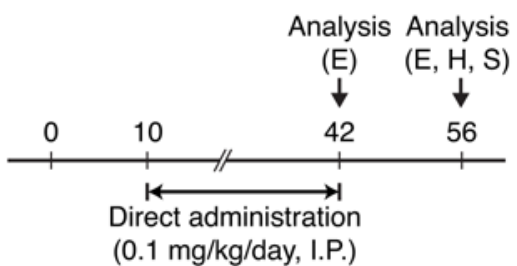

B

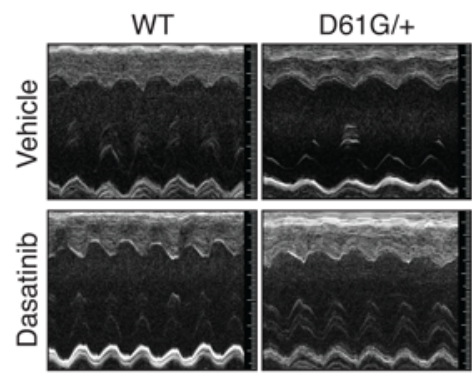

- WT $\mathrm{D} 61 \mathrm{G} /+$

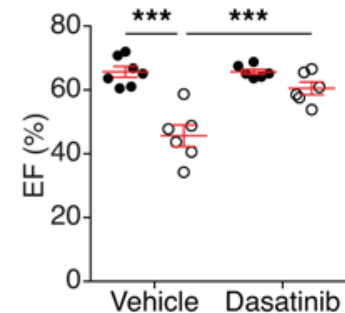

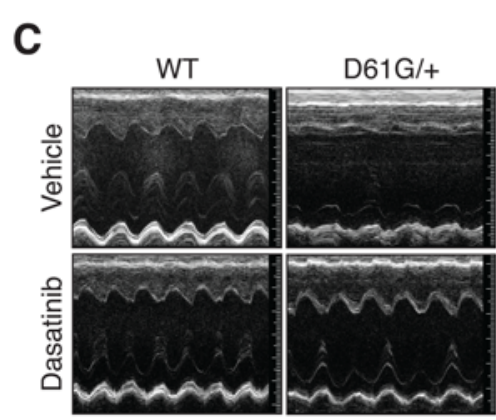
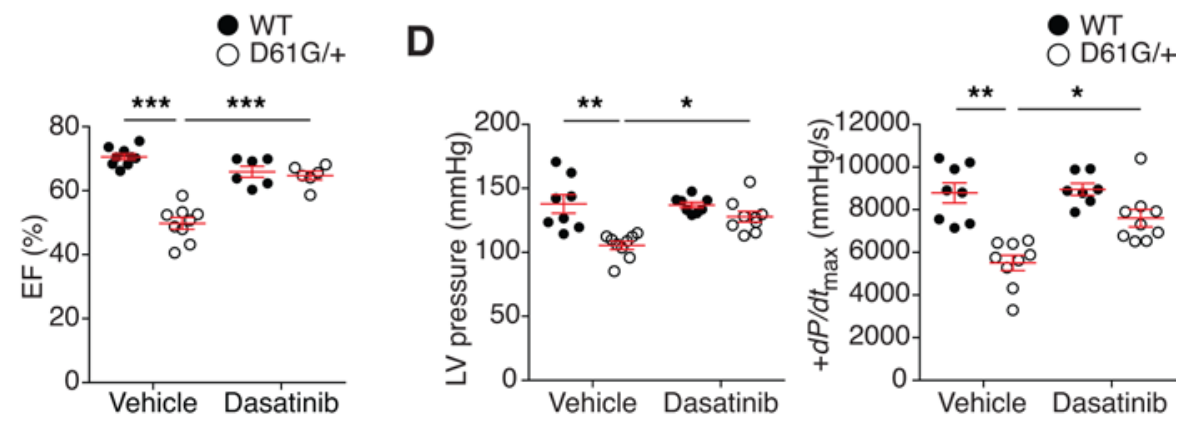

Figure 4. Low-dose dasatinib treatment rescues cardiac dysfunction of NS mice. (A) Schematic representation of postdevelopmental dasatinib treatment. WT and NS mice (D61G/+) were treated daily with vehicle or $0.1 \mathrm{mg} / \mathrm{kg}$ dasatinib (i.p.) from P10 to P42 and then treatment was discontinued for 2 weeks. Echocardiography $(E)$, invasive hemodynamic measurement $(H)$, and surgery $(S)$ were performed at the indicated time points (P42 and P56). (B) Representative echocardiographic images of vehicle- or dasatinib-treated WT and NS mice at P42. Ejection fraction (EF) percentage was measured from echocardiograms ( $n=7$ for vehicle-treated WT mice; $n=6$ for vehicle-treated NS mice and dasatinib-treated WT and NS mice). (C) Representative echocardiographic images of vehicle- or dasatinib-treated WT and NS mice at P56. EF percentage was measured from echocardiograms ( $n=9$ for vehicle-treated WT and NS mice, $n=6$ for dasatinib-treated WT and NS mice). (D) Left ventricle blood pressure (LV pressure) and the maximum rate of pressure change in the left ventricle $\left(d P / d t_{\max }\right)$ were measured in vehicle- and dasatinib-treated WT and NS mice by invasive hemodynamics ( $n=8$ for WT mouse groups, $n=9$ for NS mouse groups). Data represent the mean \pm SEM and were analyzed by 2-way ANOVA and Tukey's multiple comparison test. ${ }^{*} P<0.05 ;{ }^{* *} P<0.01 ;{ }^{* *} P<0.001$.

liver damage was detected in either WT or NS mice treated with dasatinib (Supplemental Figure 5). These results indicate that low-dose dasatinib treatment in NS mice does not result in overt toxicity and at this dose is ineffective in curtailing NS-related defects in growth, musculoskeletal development, and hematopoietic functions. However, we found that, whereas untreated NS mice had significantly reduced EF and FS levels as compared with WT untreated mice, dasatinib-treated NS mice showed comparable levels of EF as compared with WT untreated and dasatinib-treated mice (Figure 4B). Similar corrective responses in cardiac function to dasatinib in NS mice were confirmed when FS was assessed (Supplemental Table 5). Dasatinib treatment did not significantly interfere with heart rate, as there were no significant differences between WT and NS mice (Supplemental Table 5). Therefore, postnatal low-dose dasatinib treatment of NS mice can effectively ameliorate the progression toward cardiac dysfunction in these NS mice.

Next, we asked whether the effects of dasatinib on cardiac function in NS mice were sustained We reassessed cardiac function in NS mice 2 weeks after cessation of dasatinib treatment (Figure 4A). As shown, NS mice at 8 weeks of age exhibited characteristics of cardiomyopathy, as evidenced by increased heart weight to body weight and heart weight to tibia length (Supplemental Figure 6). Significant increases in LVID and a decrease in LVPW in systole corroborated the notion that these NS mice develop systolic dysfunction (Supplemental Table 6). Remarkably, a combination of echocardiography and invasive hemodynamic analyses revealed that NS mice that had been removed from dasatinib treatment for 2 weeks showed EF and FS levels that were comparable to both WT untreated and dasatinib-treated WT mice (Figure 4C and Supplemental Table 6). These changes were consistent with invasive hemodynamic measurements that showed left ventricular pressure and cardiac contractility $\left(d P / d t_{\max }\right)$ were normalized in the NS group in which dasatinib was administered for 6 weeks and then discontinued for 2 weeks (Figure 4D and Supplemental Table 7). Taken together, these data demonstrate that these NS mice exhibit features of cardiac dysfunction that are restored by postnatal low-dose dasatinib treatment. 
A
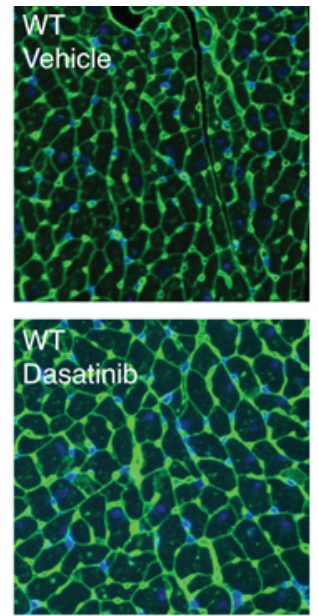
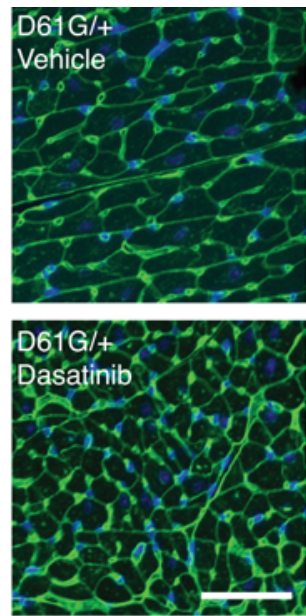
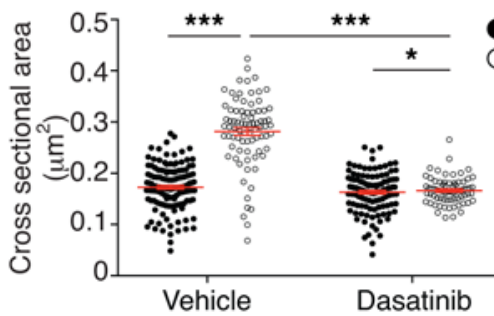

B
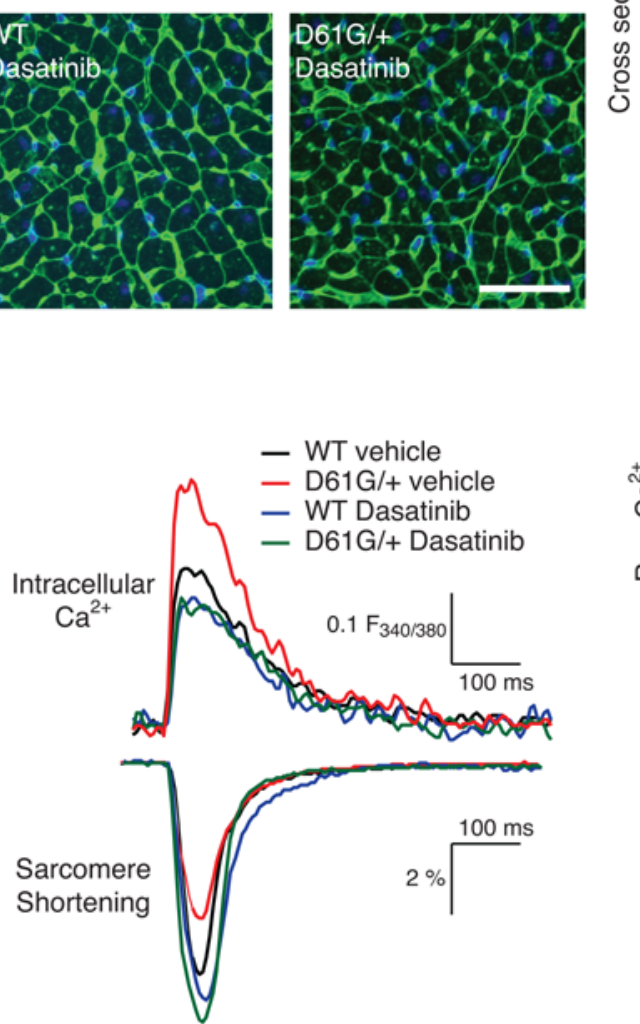

0

Vehicle

Dasatin
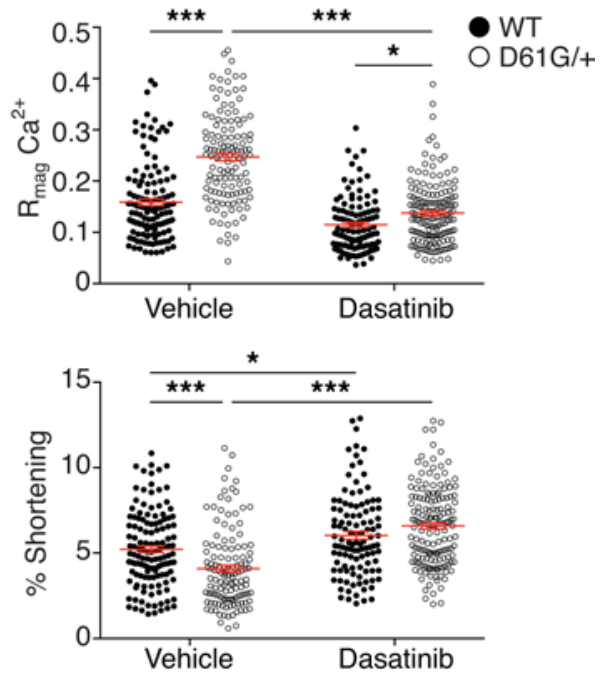

C

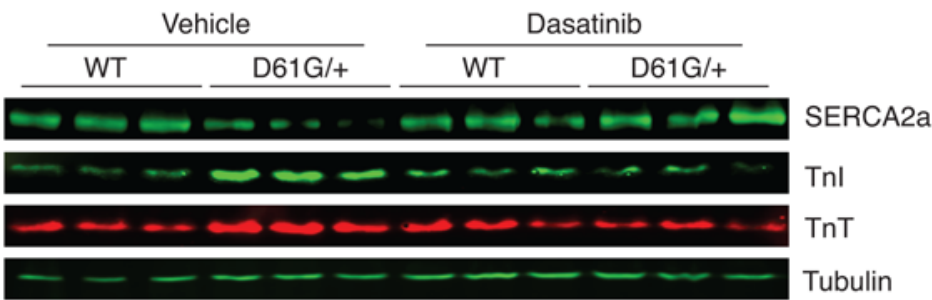

WT

D61G/+

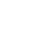

a


We measured calcium-mediated $\left(\mathrm{Ca}^{2+}\right.$-mediated) force dynamics in isolated cardiomyocytes from vehicle- and dasatinib-treated WT and NS mice. Isolated cardiomyocytes were characterized simultaneously for $\mathrm{Ca}^{2+}$ handling and contraction kinetics under electrical pacing. Cardiomyocytes from vehicle-treated NS mice showed a relative $55 \%$ higher magnitude of intracellular $\mathrm{Ca}^{2+}$ release $\left(\mathrm{R}_{\text {mag }} \mathrm{Ca}^{2+}\right)$ and delayed $\mathrm{Ca}^{2+}$ uptake compared with vehicle-treated WT cardiomyocytes (Figure 5B and Supplemental Table 8), consistent with our previous reports from NS-SHP2-overexpressing cardiomyocytes (45). Intriguingly, despite notable defects in calcium handling, there was a $22 \%$ reduction in sarcomere length shortening in vehicle-treated NS cardiomyocytes compared with cardiomyocytes from vehicle-treated WT mice (Figure 5A and Supplemental Table 8). These differences were rescued in cardiomyocytes isolated from dasatinib-treated NS mice (Figure 5B and Supplemental Table 8).

We further investigated a molecular mechanism of impaired calcium-contraction coupling. In the myocardium, sarco(endo)plasmic reticulum $\mathrm{Ca}^{2+}$-ATPase 2 (SERCA2A) is the predominant isoform responsible for $\mathrm{Ca}^{2+}$ reuptake into the sarcoplasmic reticulum between contraction events. When lysates derived from the left ventricle of vehicle-treated NS mouse hearts were immunoblotted for the expression of SERCA2A, we found a significantly decreased level of SERCA2A protein expression (Figure 5C). Consistent with the restoration of cardiac function in dasatinib-treated NS mice, cardiac tissue isolated from dasatinib-treated NS mice showed completely normalized levels of SERCA2A expression (Figure 5C). The contractile regulatory proteins troponin $\mathrm{I}(\mathrm{TnI})$ and troponin $\mathrm{T}(\mathrm{TnT})$ are often elevated in the myocardium of failing hearts. Both of these proteins were elevated in the hearts of vehicle-treated NS mice. Dasatinib treatment reversed these changes, restoring TnI and TnT levels to those of vehicle-treated WT mice (Figure 5C). These findings demonstrate that postdevelopmental treatment with dasatinib in a NS mouse model alleviates the contractile dysfunction of the myocardium.

Effects of low-dose dasatinib in Ptpn11-associated NS and NSML mice on cardiomyopathy. We hypothesize that PZR/SHP2 signaling represents a common mechanism underlying the pathogenesis of NS- and NSML-related cardiomyopathies (31). The reexpression of the fetal gene $M y h 7$ (myosin heavy chain $\beta$ ), concomitant with a reduction in Myh6 (myosin heavy chain $\alpha$ ), is a hallmark of cardiac remodeling $(46,47)$. Thus, we determined the expression of Myh7 and Myh6 genes in the hearts of NS and NSML mice. We found that Myh6 expression was significantly downregulated in vehicle-treated NS mice as compared with WT mice and dasatinib treatment resulted in an equivalent level of Myh6 expression in WT and NS mice (Figure 6A). In contrast, $M y h 7$ was prominently reexpressed in vehicle-treated NS mice, and $M y h 7$ expression was normalized to vehicle WT levels following dasatinib treatment (Figure 6A). The Myh7/Myh6 ratio confirmed that a significant shift in the expression of these cardiac myosin heavy chains occurred, indicative of cardiomyopathy in NS mice that was rescued upon dasatinib treatment (Figure 6A). The effectiveness of dasatinib to ameliorate the molecular hallmarks of cardiac dysfunction in NS mice was bolstered by the assessment of atrial natriuretic peptide (Anp) expression, which is a clinical marker of heart failure (48). Anp mRNA expression was significantly upregulated in NS mice as compared with vehicle-treated WT controls, whereas dasatinib-treated NS mice were completely rescued from the elevated mRNA expression levels of Anp (Figure 6B). To determine whether low-dose dasatinib $(0.1 \mathrm{mg} / \mathrm{kg} / \mathrm{d})$ is effective in a NSML model of cardiomyopathy, we examined the cardiac fetal gene expression from mice that express a knockin of the Ptpn11 ${ }^{Y 279 C /+}$ mutation (25). NSML mice showed significant transition from Myh6 to $M y h 7$, and dasatinib returned these values to WT levels (Figure 6C). Moreover, Anp mRNA expression in NSML mouse hearts was also significantly normalized by dasatinib treatment (Figure 6D). Collectively, these results support the conclusion that low-dose dasatinib acts to effectively rescue both NS- and NSML-mediated effects in the myocardium.

Low-dose dasatinib ameliorates cardiac fibrosis in NS and NSML mice. Histological examination of cardiac tissue revealed disorganized myofibrillar structures in the left ventricle wall of vehicle-treated NS mice (Figure 7A). In contrast, dasatinib-treated NS mice showed a profound reversal of this cardiac phenotype, in that myofibers were restored to the histological architecture similar to that of vehicle-treated WT mice (Figure 7A). Another hallmark of the cardiac dysfunction is the acquisition of cardiac fibrosis (49). Consistent with the notion that dasatinib treatment conferred protection against cardiomyopathic abnormalities in the NS heart, we found that interstitial cardiac fibrosis and the expression of Colla2 and Col3a1 genes in dasatinib-treated NS mouse hearts was markedly reduced as compared with vehicle-treated WT mice at the histological level (Figure 7, A and B). Strikingly, similar observations were made when hearts from NSML animals were assessed histologically for fibrosis and for the expression of Colla2 and Col3a1 (Figure 7, C and D). These data demonstrate that a low dose of dasatinib provides selective efficacy for preventing cardiac fibrosis in both NS and NSML mice. 
A
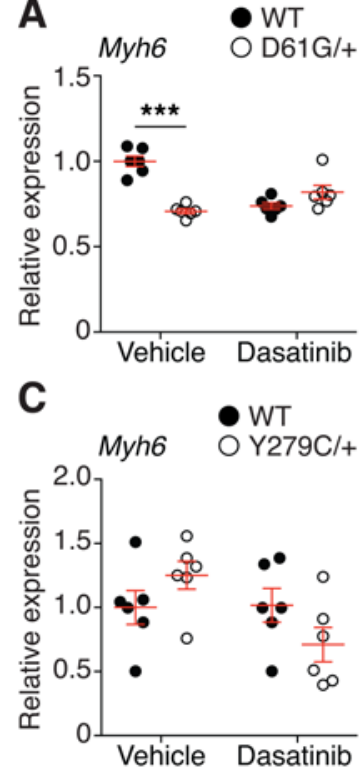
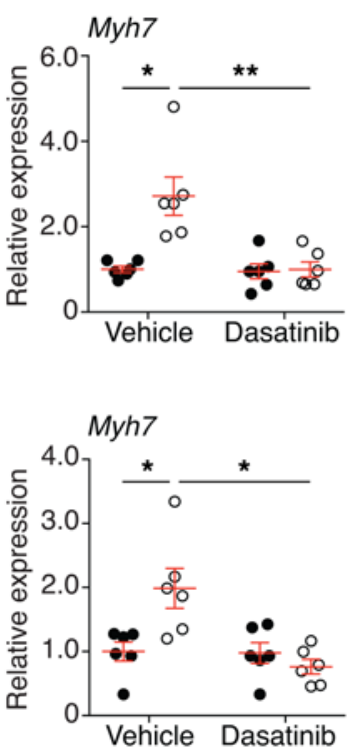
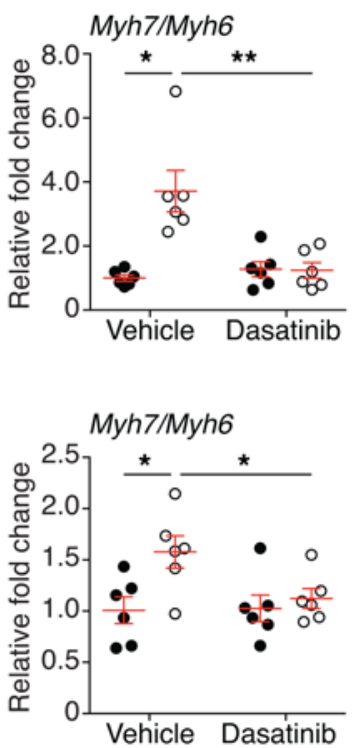

B
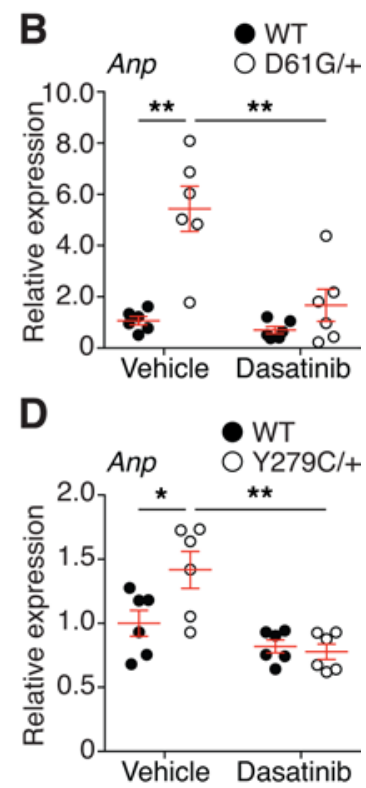

Figure 6. Low-dose dasatinib normalizes cardiac fetal gene expression in Ptpn11-associated NS and NSML mice. Total heart RNA was isolated from 8-week-old vehicle- or dasatinib-treated WT and NS (D61G/+) mice (A and B) or WT and NSML (Y279C/+) mice (C and D). Myh7, Myh6, and the ratio of Myh7 to Myh6 (A and C) and Anp (B and D) were measured by quantitative RT-PCR and normalized to the vehicle-treated WT group ( $n=6$ per group). Data represent mean \pm SEM and were analyzed by 2 -way ANOVA and Tukey's multiple comparison test. ${ }^{*} P<0.05$; ${ }^{* *} P<0.01 ;{ }^{* *} P<0.001$.

\section{Discussion}

Mouse models that represent mutations in the NS Ptpn11 allele have been shown to exhibit a range of viabilities that depend upon genetic background akin to the range of phenotypic variabilities seen among NS families (50). The variable penetrance of the Ptpn $11^{D 61 G /+}$ allele has been proposed to be an indication that modifier alleles influence phenotypic outcome (51). We found that introducing the previously reported Ptpn11 D61G allele into the B6129SF1/Tac background eliminated the previously reported lethality of this allele (38). We characterized the postdevelopmental cardiac phenotypes of this Ptpn11-associated NS mouse model and found that these mice initially presented with HCM, as defined by a significantly increased heart-weight-to-body-weight ratios, increased left ventricular wall thickness, increased ventricular mass, and reduced LVID at the age of 3 weeks that progressed to cardiac dysfunction by the age of 6 and 8 weeks. Given that NS patients with a PTPN11 mutation present with HCM less frequently than they do with PS and that in previous reports, the Ptpn11 $11^{D 61 G /+}$ allele in a different mouse background did not exhibit HCM (38), we suggest that the NS mouse model described here represents a distinct strain-specific phenotype. Indeed, it is well appreciated that genetic background can influence physiological and pathophysiological cardiovascular responses $(52,53)$. Thus, there likely exist modifier alleles that influence the penetrance of HCM that in other backgrounds appear to be either repressed and/or activated. The identity of such alleles remain unknown.

PZR has been shown to drive adhesion-dependent signaling pathways involved in cell migration and cell adhesion (36, 37). PZR is the most hyper-tyrosyl-phosphorylated protein in the hearts of NS mice, and it is also hyper-tyrosyl phosphorylated in the hearts of NSML mice (31). The ability of NS-associated mutants to propagate downstream signaling is dependent upon the engagement of its $\mathrm{SH} 2$ domains with upstream targets. PZR, when aberrantly tyrosyl phosphorylated to the extent reported in NS mice (31), likely represents a site in which aberrant SHP2 signaling is initiated. Therefore, by interfering with PZR tyrosyl phosphorylation, PZR/SHP2 binding, and/or the putative tyrosine kinase responsible for PZR phosphorylation, we speculated that NS- and NSML-associated cardiovascular outcomes might be curtailed. We used dasatinib (marketed as Sprycel), an FDA-approved chemotherapeutic drug (42) that is a tyrosine kinase inhibitor of broad specificity, as a proofof-concept strategy to test this hypothesis. In cultured fibroblasts derived from NS mice, dasatinib effectively inhibited PZR tyrosyl phosphorylation and SHP2 binding. PZR contains two major ITIMs that have been shown to serve as substrates for the Src family kinases, such as c-Src, c-Fyn, and c-Lyn as well as Csk and c-Abl (54). Although we found that dasatinib inhibited c-Src phosphorylation, these experiments do not identify 
A
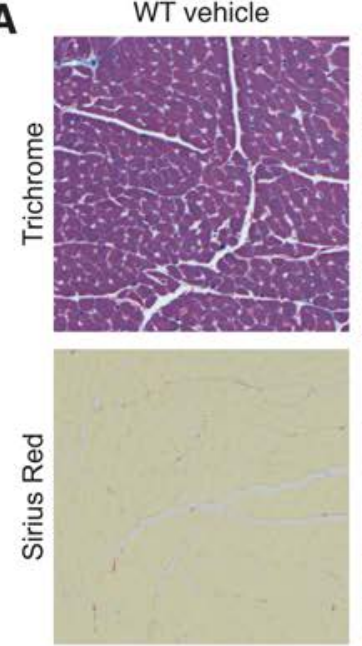

B

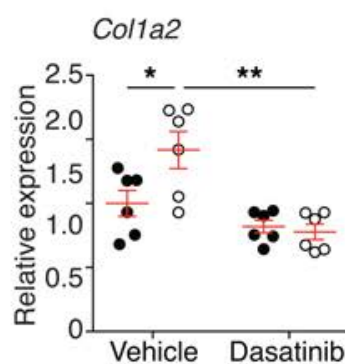

C
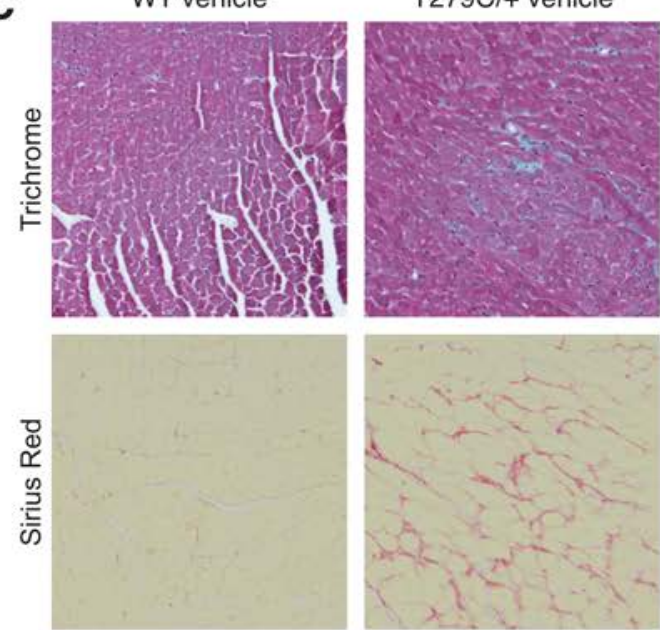

D

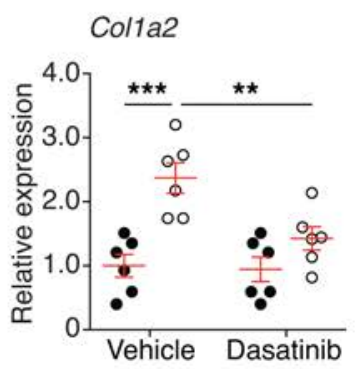

D61G/+ vehicle
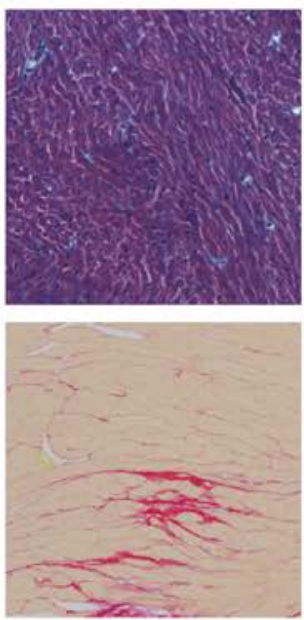

$x^{x+2-2 x}$
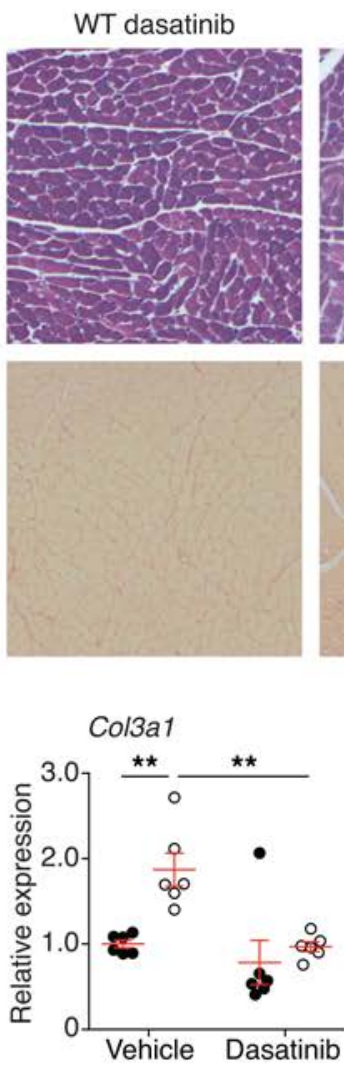

Y279C/+ vehicle
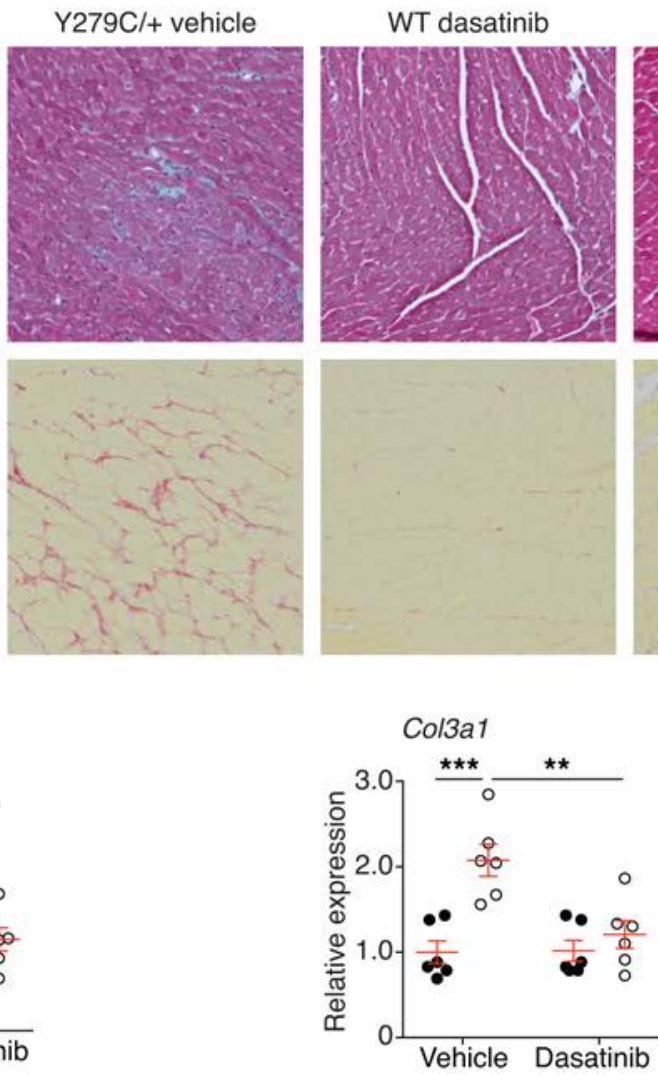

$\mathrm{D} 61 \mathrm{G} /+$ dasatinib
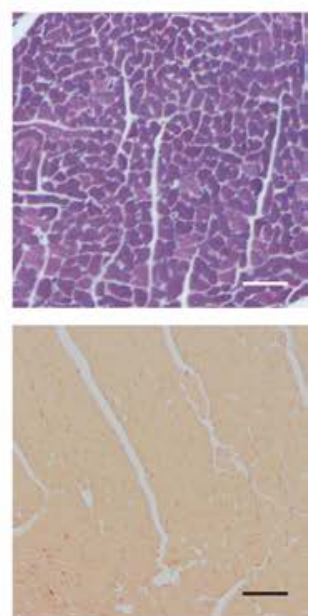

WT

D $61 \mathrm{G} /+$
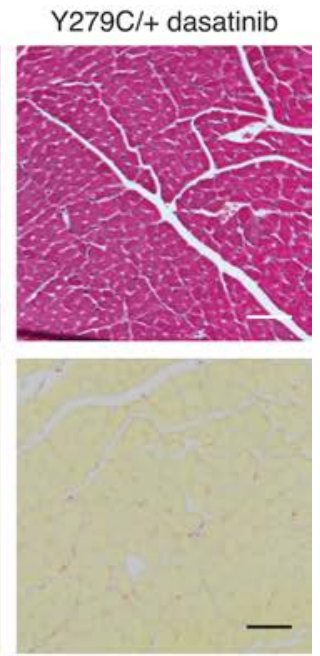

- WT

O $279 \mathrm{C} /+$

\section{.}

been leukemia, has been reported to have cardiotoxic effects (42). At higher concentrations in utero, we did note more generalized toxicity and lethality. However, at $0.1 \mathrm{mg} / \mathrm{kg} / \mathrm{d}$ doses, the effects of dasatinib were well tolerated to the extent that other NS-related presentations, such as growth retardation, facial dysmorphism, and splenomegaly, were unaffected. Thus, the effects of dasatinib at this low dose appear to be selective to the heart. 
Assessment of echocardiography parameters in NS mice administered low-dose dasatinib either during development or postnatally showed a marked improvement in heart function, as assessed by increases in EF and FS as compared with vehicle-treated NS mice. Postnatal low-dose dasatinib treatment of NS mice also ameliorated the progression of cardiac fibrosis. Low-dose dasatinib treatment either in utero or postnatally reversed the presentation of cardiac dysfunction, suggesting that the activity of a tyrosine kinase that mediates PZR and/or other targets plays a critical role in NS cardiac disease progression. The precise identity of this tyrosine kinase(s) remains to be determined.

Previous reports have indicated that NS-associated SHP2 mutants can enhance $\mathrm{Ca}^{2+}$ signaling in cardiomyocytes (45). Here, we showed that calcium-mediated contractile properties are impaired in NS cardiomyocytes. Given that $\mathrm{Ca}^{2+}$-mediated contractile properties are fundamental to the function of the force-generating properties of the myocyte, our observations that cardiomyocytes from NS mice exhibit dysfunctional $\mathrm{Ca}^{2+}$ handling and force generation strongly support the supposition that these mice present features of cardiac dysfunction, at minimum due to defects in cardiomyocyte contractility and/or secondary consequences of left ventricular remodeling. Postnatal low-dose dasatinib treatment restored $\mathrm{Ca}^{2+}$-mediated contraction. Of note, NS mice showed enhanced intracellular $\mathrm{Ca}^{2+}$ accumulation yet impaired sarcomere shortening as compared with WT cardiomyocytes. These results suggest the NS mutant uncouples the ability of increased $\mathrm{Ca}^{2+}$ to drive sarcomeric force, implying that some level of desensitization to one or more $\mathrm{Ca}^{2+}$-responsive targets may occur. At this juncture, we cannot ascribe a precise pathway delineating how PTPN11 mutations cause a loss of $\mathrm{Ca}^{2+}$-mediated contraction coupling. However, it has been reported that NS and NSML mutants can affect $\mathrm{Ca}^{2+}$ transients in the myocardium $(45,55)$. We were able to demonstrate that SERCA2A was reduced in its level of expression in NS mice, and this too was restored to WT levels in postnatal low-dose dasatinib-treated NS mice. Although these data suggest that the site action of dasatinib occurs within the myocardium consistent with other reports (56), we cannot exclude the contribution of other cell types, such as endocardial cells, which have been shown to be the site of action responsible for the manifestation of the NS phenotype in mice (51). Regardless, our data support the involvement of the myocardium as responsible for the improved contractile properties following dasatinib administration.

We show that the molecular parameters of cardiac fetal gene expression that represent characteristics of HCM were rescued upon treatment with low doses of dasatinib in both NS and NSML mice. The expression levels of Anp were also restored to WT vehicle-treated levels. Collectively, these data argue for the correction of the HCM phenotype molecularly by dasatinib. Given that NS and NSML represent opposing activities at the level of SHP2 catalysis, our results imply a mechanism whereby interference with PZR tyrosyl phosphorylation and/or dasatinib-sensitive tyrosine kinase activity is sufficient to correct the molecular parameters of HCM. Previous reports have been conducted using inhibitors that target the Ras/ MAPK pathway in order to ameliorate NS- and NSML-mediated HCM. For example, a MEK inhibitor has been shown to ameliorate the cardiac defects in NS-specific $K$-Ras ${ }^{V 14 I_{-}}$, Raf1 R613V $_{-}$, and Sos $1^{E 846 K_{-}}$expressing mice (28-30). MEK/ERK inhibition has also been shown to rescue the craniofacial defects in Ptpn11079R mice and cognitive defects in Ptpn11 ${ }^{D 61 G}$ mice $(39,57)$. Similarly, NSML mouse models that present with HCM have been reported to respond to inhibition of the mTOR pathway using rapamycin (25). Clinical studies also have reported that treatment with a rapamycin analog in a NSML patient improved cardiomyopathy and decreased brain natriuretic peptide levels in serum (26). Whereas treating the cardiac defects for NS or NSML might require distinct therapeutic regimens, our data suggest that in conditions in which either NS- or NSML-related cardiac dysfunction is suspected, a single therapeutic such as dasatinib might provide a novel strategy to improve cardiac functionality. Further studies are required using more specific tyrosine kinase inhibitors at low doses both in other RASopathy models and in adult models of cardiac failure to determine if this strategy has more broad effectiveness in CHD. Indeed, a recent report suggests that low-dose dasatinib in an adult mouse model of cardiac dysfunction curtails the development of fibrosis and improves left ventricular function (56).

In summary, we identify PZR/SHP2 as a targetable signaling node that is associated with the progression of NS and NSML. Our findings further suggest that a short-course low-dose treatment of children at risk for the cardiac pathologies of NS and NSML, and potentially other RASopathies, might curtail the development of significant HCM and cardiac dysfunction in these children. Success in preventing or attenuating HCM in NS and NSML might provide a framework for more targeted pharmacological approaches to treating NS-related cardiomyopathies. 


\section{Methods}

Antibodies, chemicals, cell lines, and plasmids. The following antibodies were used either for immunoblotting (IB) or immunoprecipitation (IP) as indicated. Rabbit polyclonal SHP2 (sc-280, IB 1:1,000, IP 1:100) antibody was from Santa Cruz Biotechnology. Rabbit monoclonal phospho-PZR (8181, Y241, clone D6F9, IB 1:1,000), rabbit monoclonal phospho-PZR (8088, Y263, clone D6A5, IB 1:1,000), rabbit polyclonal phospho-Src (2101, Y416, IB 1:1,000), mouse monoclonal c-Src (2110, clone L4A1, IB 1:1,000), rabbit polyclonal phospho-ERK1/2 (T202/Y204; 9101, IB 1:1,000), mouse monoclonal ERK (9107, clone 3A7, IB 1:1,000), rabbit polyclonal SERCA2A (9580, IB 1:1,000), and rabbit polyclonal TnI (4002, IB 1:1,000) antibodies were purchased from Cell Signaling. Rabbit polyclonal $\alpha$ tubulin (ab4074, IB 1:1,000) antibodies were obtained from Abcam. Mouse monoclonal SHP2 (610622, clone 79, IB 1:1,000, IP 1:100) antibody was purchased from BD Biosciences. Rabbit polyclonal TnT (MS-295, IB 1:1,000) was from Thermo Scientific. Mouse monoclonal phosphotyrosine (05-321, clone 4G10, IB 1:1,000) antibody was purchased from Millipore. Rabbit polyclonal PZR antibody (IB 1:1,000) was provided by Z.J. Zhao (University of Oklahoma, Oklahoma City, Oklahoma, USA) (34). Dasatinib was purchased from Biovision. The alanine aminotransferase activity assay kit was purchased from Cayman Chemical. The mouse embryonic fibroblasts were isolated from WT and Ptpn11 $1^{D 61 G /+}$ mice as described previously (32).

$I P$ and IB. Cells or heart tissue were lysed on ice in lysis buffer (25 mM Tris-HCl, pH 7.4, $136 \mathrm{mM}$ $\mathrm{NaCl}, 1 \mathrm{mM} \mathrm{CaCl}_{2}, 1 \mathrm{mM} \mathrm{MgCl} 2,1 \%$ Nonidet P-40, 1 mM Na $\mathrm{VO}_{4}, 10 \mathrm{mM} \mathrm{NaF}, 1 \mathrm{mM}$ benzamidine, 1 $\mathrm{mM}$ PMSF, $1 \mu \mathrm{g} / \mathrm{ml}$ pepstatin A, $5 \mu \mathrm{g} / \mathrm{ml}$ aprotinin, and $5 \mu \mathrm{g} / \mathrm{ml}$ leupeptin). Cell or tissue lysates were incubated at $4^{\circ} \mathrm{C}$ for 30 minutes and clarified by centrifugation at $20,000 \mathrm{~g}$ at $4^{\circ} \mathrm{C}$ for 10 minutes. Protein concentration was determined using the BCA reagent according to the manufacturer's instructions (Pierce). For IP, $500 \mu \mathrm{g}$ of lysate was incubated with $1 \mu \mathrm{g}$ of the indicated antibody at $4^{\circ} \mathrm{C}$ overnight. Immune complexes were collected on protein A-Sepharose beads for 4 hours at $4^{\circ} \mathrm{C}$, washed 3 times with lysis buffer, and heated to $95^{\circ} \mathrm{C}$ in sample buffer for 5 minutes. Total lysates or immune complexes were subjected to SDS-PAGE and IB. The sites of antibody binding were visualized using either enhanced chemiluminescence detection or the Odyssey Imaging System.

Animal handling. Ptpn $11^{D 61 G /+}$ mice were provided by Benjamin Neel (New York University, New York, New York, USA), and Ptpn11 ${ }^{Y 279 C /+}$ mice were provided by Maria Kontaridis (Beth Israel Deaconess Medical Center, Boston, Massachusetts, USA). Mice were genotyped as described previously $(25,38)$. Ptpn11 ${ }^{\text {D61G/+ }}$ male mice were crossed with WT B6129SF1/Tac female mice, and their offspring were genotyped by PCR and digestion with AgeI for the D61G allele. Ptpn11 $1^{1279 C /+}$ male mice were crossed with WT C57BL/6J females, and offspring were genotyped by PCR for the Y279C allele. Dasatinib (Biovision) was suspended in vehicle (1\% DMSO in citrate buffer). For prenatal treatment, dasatinib was injected i.p. (0.1 $\mathrm{mg} / \mathrm{kg}$ body weight) into pregnant mice daily, beginning on gestational day 7.5 and continuing (in nursing females) until P9. Vehicle-injected mice served as control. Beginning at P10, dasatinib or vehicle alone was injected (i.p.) directly into pups daily until 8 weeks after birth. For postnatal treatment, dasatinib was injected (i.p.) into pups at P10 until 6 weeks after birth; injection was discontinued for 2 weeks.

Histology. Heart, liver, and spleen were isolated from vehicle- or dasatinib-treated WT, NS, and NSML mice. Tissues were fixed in $4 \%$ paraformaldehyde in PBS, processed for paraffin sections, and stained with H\&E, Masson's trichrome, Picrosirius red, and Alexa488-conjugated wheat germ agglutinin. Tissue images were obtained under bright-field microscope (Olympus BX51) or epifluorescence microscope (Zeiss Axiovert 200) at the Yale Liver Center.

Echocardiography. Mice were anesthetized in a sealed plastic chamber with $1 \%$ isoflurane in oxygen until immobile and then were transferred onto a heated procedure board $\left(37^{\circ} \mathrm{C}\right)$. Animals were kept anesthetized with $1 \%$ isoflurane supplied by a nose cone connected to the isoflurane vaporizer during the entire procedure. The scan head was placed on the chest of the mouse, and stable image signals (both B mode and $\mathrm{M}$ mode) were acquired and data analyzed was with Vevo 770 (VisualSonics). Systolic and diastolic left ventricle peripheral wall thickness, chamber diameter, and interventricular wall thickness were measured with M mode image. Percentages of EF and FS were calculated.

Hemodynamic studies. Anesthesia was induced by i.p. injection of ketamine $(100 \mathrm{mg} / \mathrm{kg})$ and xylazine $(5 \mathrm{mg} / \mathrm{kg})$. The animal was placed on a warm pad and an incision was made on the neck. The right-side carotid artery was exposed, and a 1.9-French transducer-tipped catheter (Millar Inc.) was inserted into the artery and then was advanced into the left ventricle. Left ventricular pressures, including high-fidelity 
positive, negative $d p / d t$, and heart rate were measured under basal conditions. Data were recorded and analyzed by using LabChart software.

$R N A$ extraction and quantitative real-time PCR analysis. RNA was isolated from mouse hearts using an RNeasy kit (Qiagen) according to the manufacturer's instructions. A total of $1 \mu \mathrm{g}$ RNA was reverse transcribed to generate cDNA using a reverse-transcriptase PCR kit (Applied Biosystems). Realtime quantitative PCR was carried out in triplicate using the Applied Biosystems 7500 Fast Real-Time PCR system and SYBR green gene expression master mix with following primer pairs: 18S rRNA, 5'-ACCGCAGCTAGGAATAATGGA-3'， 5'-ACCAAAAGCCTTGACTCCG-3'; Anp, 5'-CCTGGAGGAGAAGATGCCGGTAGAA-3'， 5'-CCCCAGTCCAGGGAGGCACCTCGG-3'; Myh6, 5'-GTCCCGGACACTGGACCAGGCC-3', 5'-CTCCTTTTCTTCCAGTTGCCTAGCCAA-3'; Myh7, 5'-GAGCAAGGCCGAGGAGACGCAGCGT-3'， 5'-GAGCCTCCTTCTCGTCCAGCTGCCGG-3'; Colla2, 5'-AGGTCTTCCTGGAGCTGATG-3', 5'-ACCCACAGGGCCTTCTTTAC-3'; and Col3a1, 5'-ACAGCAAATTCACTTACACAGTTC-3', 5'-CTCATTGCCTTGCGTGTTT-3'. All relative gene expression levels were analyzed using the $\Delta \mathrm{C}_{\mathrm{T}}$ method and normalized to $18 \mathrm{~S}$ rRNA expression.

Enzymatic digestion of cardiac tissue for single-cell analysis. Cardiomyocytes from 8-week-old mice were isolated by a modified Langendorff procedure (58). In brief, the hearts were quickly excised and cannulated to a Langendorff apparatus where they were perfused with $37^{\circ} \mathrm{C} \mathrm{Ca}^{2+}$-free perfusion buffer (in $25 \mathrm{mM}$ HEPES, $118 \mathrm{mM} \mathrm{NaCl}, 4.8 \mathrm{mM} \mathrm{KCl}, 2.0 \mathrm{mM} \mathrm{KH}_{2} \mathrm{PO}_{4}, 2.55 \mathrm{mM} \mathrm{MgSO}_{4}, 10 \mathrm{mM}$ 2,3-butanedione 2-monoxime, and $10 \mathrm{mM}$ glucose). To digest the tissue, the heart was perfused with buffer containing $0.5 \mathrm{mg} / \mathrm{ml}$ Liberase DH (Roche Applied Science). After approximately 10 minutes, the heart was removed from the Langendorff apparatus, and the right ventricle and atria were removed. The left ventricle was isolated, cut into small pieces, and digested further at $37^{\circ} \mathrm{C}$ in digestion solution with mechanical agitation for 5 to 10 minutes and then gently triturated to liberate individual cells. Remaining tissue chunks were transferred to fresh digestion buffer, and the process was repeated up to 6 times or until all tissue was digested. Cells were removed from collagenase by gentle centrifugation and resuspended in several washing steps of buffer-containing FBS and gradually reintroduced to $\mathrm{Ca}^{2+}(0.05-1.1 \mathrm{mM})$ through step-wise additions of concentrated $\mathrm{CaCl}_{2}$ solution. Cells were allowed to rest for at least 1 hour before imaging.

Functional characterization of cardiomyocytes. Cardiomyocytes were imaged in Tyrodes solution (140 mM $\mathrm{NaCl}, 5.4 \mathrm{mM} \mathrm{KCl}, 1.8 \mathrm{mM} \mathrm{CaCl}_{2}, 1 \mathrm{mM} \mathrm{MgCl}_{2}, 25 \mathrm{mM}$ HEPES, and $10 \mathrm{mM}$ glucose). Cell pellets were loaded for 15 minutes in the dark with Tyrodes supplemented with $2.5 \mu \mathrm{M}$ Fura- $2 \mathrm{AM}$, supplemented with pluronic acid $(20 \% \mathrm{w} / \mathrm{v})$ for $\mathrm{Ca}^{2+}$ fluorescence imaging. After 15 minutes of loading, the cells were resuspended in fresh Tyrodes solution and allowed to settle until imaging. Cardiomyocyte $\mathrm{Ca}^{2+}$ transients and unloaded shortening contractions were measured using an inverted microscope (Nikon Eclipse) equipped with a temperature-controlled perfusion bath (Cell MicroControls) under constant perfusion of $37^{\circ} \mathrm{C}$ Tyrodes solution. Cells were field stimulated at $1 \mathrm{~Hz}$. Contractile events were imaged in real time using a sarcomere length camera system (HVSL, Aurora Scientific). Only rod-shaped cells with well-defined sarcomere striations that contracted when stimulated were selected for measurement. Sarcomere length was measured and recorded for 10 consecutive beats and subsequently averaged across beats to produce a single waveform. Calcium transient measurements were recorded simultaneously using alternating excitation wavelengths of 340 and $380 \mathrm{~nm}$ generated at an overall rate of $100 \mathrm{~Hz}$ by a RatioMaster fluorescence system (PTI). Fluorescence emission was filtered at a center wavelength of $510 \mathrm{~nm}$ and quantified to obtain responses to the alternating excitation wavelengths $\left(\mathrm{F}_{340}\right.$ and $\mathrm{F}_{380}$, respectively). $\mathrm{Ca}^{2+}$ transients were reported as the interpolated ratio of the two fluorescence intensities $\left(\mathrm{F}_{340} / \mathrm{F}_{380}\right)$ at each time point. Data were recorded using a DAP5216a data acquisition system (Microstar Laboratories) and processed using custom software written in MATLAB (MathWorks). Peak sarcomere length shortening (peak SL shortening), time to peak shortening (TTP), time to 50\% relengthening (RT50), the magnitude of the calcium transient $\left(\mathrm{Ca}^{2+}\right.$ $\mathrm{R}_{\text {mag }}=\operatorname{Max} \mathrm{F}_{340} / \mathrm{F}_{380}-\mathrm{Min}_{340} / \mathrm{F}_{380}$ ), and the rate of calcium decay, Tau ( $\mathrm{Tau}_{\mathrm{Ca} 2+}$ ), were calculated.

Statistics. The number of samples $(n)$ used in each experiment is shown. All in vitro experiments were performed at least 3 times independently. Sample size for animal studies was not estimated, and samples were randomized. The investigators were blinded during echocardiography, hemodynamics and cardiomyocyte contraction coupling experiments, and outcome assessment. Statistical analysis and graphing were performed using GraphPad Prism 6 software. We did not estimate variations in the data. All data represent the mean \pm SEM. For $P$ value determinations, we used unpaired Student's $t$ test or 1-way or 2-way ANOVA was used with Tukey's multiple comparison. 
Study approval. All procedures, animal studies, and animal handling were approved by the Institutional Animal Care and Use Committee at Yale University.

\section{Author contributions}

JSY and AMB conceptualized the research, designed the experiments, and wrote the manuscript. JSY performed biochemical and histological experiments and analyzed data. JSY and YH performed echocardiography and hemodynamic studies. JSY and IYK performed cardiomyocyte isolation. ATK performed cardiomyocyte contraction coupling experiments. BEE, SGC, and FJG discussed the results of experiments and edited the manuscript.

\section{Acknowledgments}

This work was supported by NIH R01 grants GM099801 and AR066003 and an American Heart Association grant (16GRNT27250122) to AMB.

Address correspondence to: Anton M. Bennett, Yale University School of Medicine, Department of Pharmacology, SHM B226D, 333 Cedar Street, New Haven, Connecticut 06520-8066, USA. Phone: 203.737.2441; E-mail: anton.bennett@yale.edu.

1. Romano AA, et al. Noonan syndrome: clinical features, diagnosis, and management guidelines. Pediatrics. 2010;126(4):746-759.

2. Tartaglia M, Gelb BD, Zenker M. Noonan syndrome and clinically related disorders. Best Pract Res Clin Endocrinol Metab. 2011;25(1):161-179

3. Tartaglia M, Gelb BD. Noonan syndrome and related disorders: genetics and pathogenesis. Annu Rev Genomics Hum Genet 2005;6:45-68

4. Tartaglia M, et al. Mutations in PTPN11, encoding the protein tyrosine phosphatase SHP-2, cause Noonan syndrome. Nat Genet. 2001;29(4):465-468.

5. Tartaglia M, et al. PTPN11 mutations in Noonan syndrome: molecular spectrum, genotype-phenotype correlation, and phenotypic heterogeneity. Am J Hum Genet. 2002;70(6):1555-1563.

6. Kurose A, Oyama K, Murakami Y, Ohyama K, Segawa I, Sawai T. Dilated cardiomyopathy in Noonan's syndrome: a first autopsy case. Hum Pathol. 2000;31(6):764-767.

7. Yu CM, Chow LT, Sanderson JE. Dilated cardiomyopathy in Noonan's syndrome. Int J Cardiol. 1996;56(1):83-85

8. Wilmshurst PT, Katritsis D. Restrictive and hypertrophic cardiomyopathies in Noonan syndrome: the overlap syndromes. Heart. 1996;75(1):94-97.

9. Cooke RA, Chambers JB, Curry PV. Noonan's cardiomyopathy: a non-hypertrophic variant. Br Heart J. 1994;71(6):561-565.

10. Colquitt JL, Noonan JA. Cardiac findings in Noonan syndrome on long-term follow-up. Congenit Heart Dis. 2014;9(2):144-150.

11. Shimizu A, Oku Y, Matsuo K, Hashiba K. Hypertrophic cardiomyopathy progressing to a dilated cardiomyopathy-like feature in Noonan's syndrome. Am Heart J. 1992;123(3):814-816.

12. Shaw AC, Kalidas K, Crosby AH, Jeffery S, Patton MA. The natural history of Noonan syndrome: a long-term follow-up study Arch Dis Child. 2007;92(2):128-132.

13. Digilio MC, et al. RASopathies: Clinical Diagnosis in the First Year of Life. Mol Syndromol. 2011;1(6):282-289.

14. Edwards JJ, et al. A PTPN11 allele encoding a catalytically impaired SHP2 protein in a patient with a Noonan syndrome phenotype. Am J Med Genet A. 2014;164A(9):2351-2355.

15. Tajan M, de Rocca Serra A, Valet P, Edouard T, Yart A. SHP2 sails from physiology to pathology. Eur J Med Genet. 2015;58(10):509-525.

16. Hof P, Pluskey S, Dhe-Paganon S, Eck MJ, Shoelson SE. Crystal structure of the tyrosine phosphatase SHP-2. Cell. 1998;92(4):441-450.

17. Lechleider R, et al. Activation of the SH2-containing phosphotyrosine phosphatase SH-PTP2 by its binding site, phosphotyrosine 1009, on the human platelet-derived growth factor receptor $\beta$. J Biol Chem. 1993;268(29):21478-21481.

18. Mohi MG, et al. Prognostic, therapeutic, and mechanistic implications of a mouse model of leukemia evoked by Shp2 (PTPN11) mutations. Cancer Cell. 2005;7(2):179-191.

19. O'Reilly AM, Neel BG. Structural determinants of SHP-2 function and specificity in Xenopus mesoderm induction. Mol Cell Biol. 1998;18(1):161-177.

20. Qiu W, et al. Structural insights into Noonan/LEOPARD syndrome-related mutants of protein-tyrosine phosphatase SHP2 (PTPN11). BMC Struct Biol. 2014;14:10.

21. Yu ZH, et al. Molecular basis of gain-of-function LEOPARD syndrome-associated SHP2 mutations. Biochemistry. 2014;53(25):4136-4151.

22. Bennett AM, Tang TL, Sugimoto S, Walsh CT, Neel BG. Protein-tyrosine-phosphatase SHPTP2 couples platelet-derived growth factor receptor beta to Ras. Proc Natl Acad Sci U S A. 1994;91(15):7335-7339.

23. Stewart RA, et al. Phosphatase-dependent and -independent functions of Shp2 in neural crest cells underlie LEOPARD syndrome pathogenesis. Dev Cell. 2010;18(5):750-762.

24. Korf B, et al. The third international meeting on genetic disorders in the RAS/MAPK pathway: towards a therapeutic approach. Am J Med Genet A. 2015;167A(8):1741-1746.

25. Marin TM, et al. Rapamycin reverses hypertrophic cardiomyopathy in a mouse model of LEOPARD syndrome-associated 
PTPN11 mutation. J Clin Invest. 2011;121(3):1026-1043.

26. Hahn A, et al. Rapidly progressive hypertrophic cardiomyopathy in an infant with Noonan syndrome with multiple lentigines: palliative treatment with a rapamycin analog. Am J Med Genet A. 2015;167A(4):744-751.

27. Nakamura T, et al. Mediating ERK $1 / 2$ signaling rescues congenital heart defects in a mouse model of Noonan syndrome. $J$ Clin Invest. 2007;117(8):2123-2132.

28. Wu X, et al. MEK-ERK pathway modulation ameliorates disease phenotypes in a mouse model of Noonan syndrome associated with the Raf1(L613V) mutation. J Clin Invest. 2011;121(3):1009-1025.

29. Chen PC, et al. Activation of multiple signaling pathways causes developmental defects in mice with a Noonan syndrome-associated Sos1 mutation. J Clin Invest. 2010;120(12):4353-4365.

30. Hernández-Porras I, et al. K-RasV14I recapitulates Noonan syndrome in mice. Proc Natl Acad Sci USA. 2014;111(46):16395-16400.

31. Paardekooper Overman J, et al. PZR coordinates Shp2 Noonan and LEOPARD syndrome signaling in zebrafish and mice. Mol Cell Biol. 2014;34(15):2874-2889.

32. Eminaga S, Bennett AM. Noonan syndrome-associated SHP-2/Ptpn11 mutants enhance SIRPalpha and PZR tyrosyl phosphorylation and promote adhesion-mediated ERK activation. J Biol Chem. 2008;283(22):15328-15338.

33. Zhao R, Zhao ZJ. Dissecting the interaction of SHP-2 with PZR, an immunoglobulin family protein containing immunoreceptor tyrosine-based inhibitory motifs. J Biol Chem. 2000;275(8):5453-5459.

34. Zhao ZJ, Zhao R. Purification and cloning of PZR, a binding protein and putative physiological substrate of tyrosine phosphatase SHP-2. J Biol Chem. 1998;273(45):29367-29372.

35. Zhao R, Guerrah A, Tang H, Zhao ZJ. Cell surface glycoprotein PZR is a major mediator of concanavalin A-induced cell signaling. J Biol Chem. 2002;277(10):7882-7888.

36. Zannettino AC, et al. Novel mesenchymal and haematopoietic cell isoforms of the SHP-2 docking receptor, PZR: identification, molecular cloning and effects on cell migration. Biochem J. 2003;370(Pt 2):537-549.

37. Roubelakis MG, Martin-Rendon E, Tsaknakis G, Stavropoulos A, Watt SM. The murine ortholog of the SHP-2 binding molecule, PZR accelerates cell migration on fibronectin and is expressed in early embryo formation. J Cell Biochem. 2007;102(4):955-969.

38. Araki T, et al. Mouse model of Noonan syndrome reveals cell type- and gene dosage-dependent effects of Ptpn11 mutation. Nat Med. 2004;10(8):849-857.

39. Lee YS, et al. Mechanism and treatment for learning and memory deficits in mouse models of Noonan syndrome. Nat Neurosci. 2014;17(12):1736-1743.

40. Keilhack H, David FS, McGregor M, Cantley LC, Neel BG. Diverse biochemical properties of Shp2 mutants. Implications for disease phenotypes. J Biol Chem. 2005;280(35):30984-30993.

41. Jia D, et al. Amplification of MPZL1/PZR promotes tumor cell migration through Src-mediated phosphorylation of cortactin in hepatocellular carcinoma. Cell Res. 2014;24(2):204-217.

42. Aguilera DG, Tsimberidou AM. Dasatinib in chronic myeloid leukemia: a review. Ther Clin Risk Manag. 2009;5(2):281-289.

43. Lin AE, et al. Adults with genetic syndromes and cardiovascular abnormalities: clinical history and management. Genet Med. 2008;10(7):469-494.

44. Nishikawa T, Ishiyama S, Shimojo T, Takeda K, Kasajima T, Momma K. Hypertrophic cardiomyopathy in Noonan syndrome. Acta Paediatr Jpn. 1996;38(1):91-98.

45. Uhlén P, Burch PM, Zito CI, Estrada M, Ehrlich BE, Bennett AM. Gain-of-function/Noonan syndrome SHP-2/Ptpn11 mutants enhance calcium oscillations and impair NFAT signaling. Proc Natl Acad Sci U S A. 2006;103(7):2160-2165.

46. Schoenfeld JR, et al. Distinct molecular phenotypes in murine cardiac muscle development, growth, and hypertrophy. $J$ Mol Cell Cardiol. 1998;30(11):2269-2280.

47. Morita H, Seidman J, Seidman CE. Genetic causes of human heart failure. J Clin Invest. 2005;115(3):518-526.

48. Burnett JC, et al. Atrial natriuretic peptide elevation in congestive heart failure in the human. Science. 1986;231(4742):1145-1147.

49. Weber KT, Brilla CG. Pathological hypertrophy and cardiac interstitium. Fibrosis and renin-angiotensin-aldosterone system. Circulation. 1991;83(6):1849-1865.

50. Gelb BD, Tartaglia M. Noonan syndrome and related disorders: dysregulated RAS-mitogen activated protein kinase signal transduction. Hum Mol Genet. 2006;15 Spec No 2:R220-R226.

51. Araki T, Chan G, Newbigging S, Morikawa L, Bronson RT, Neel BG. Noonan syndrome cardiac defects are caused by PTPN11 acting in endocardium to enhance endocardial-mesenchymal transformation. Proc Natl Acad Sci U S A. 2009;106(12):4736-4741.

52. Deschepper CF, Olson JL, Otis M, Gallo-Payet N. Characterization of blood pressure and morphological traits in cardiovascular-related organs in 13 different inbred mouse strains. J Appl Physiol. 2004;97(1):369-376.

53. Barrick CJ, Rojas M, Schoonhoven R, Smyth SS, Threadgill DW. Cardiac response to pressure overload in 129S1/SvImJ and C57BL/6J mice: temporal- and background-dependent development of concentric left ventricular hypertrophy. Am JPhysiol Heart Circ Physiol. 2007;292(5):H2119-H2130.

54. Kusano K, Thomas TN, Fujiwara K. Phosphorylation and localization of protein-zero related (PZR) in cultured endothelial cells. Endothelium. 2008;15(3):127-136.

55. Clay SA, Domeier TL, Hanft LM, McDonald KS, Krenz M. Elevated Ca2+ transients and increased myofibrillar power generation cause cardiac hypercontractility in a model of Noonan syndrome with multiple lentigines. Am J Physiol Heart Circ Physiol. 2015;308(9):H1086-H1095.

56. Balasubramanian S, et al. Dasatinib attenuates pressure overload induced cardiac fibrosis in a murine transverse aortic constriction model. PLoS One. 2015;10(10):e0140273.

57. Nakamura T, Gulick J, Pratt R, Robbins J. Noonan syndrome is associated with enhanced pERK activity, the repression of which can prevent craniofacial malformations. Proc Natl Acad Sci USA. 2009;106(36):15436-15441.

58. Xu X, Colecraft HM. Primary culture of adult rat heart myocytes. J Vis Exp. 2009;28:e1308. 